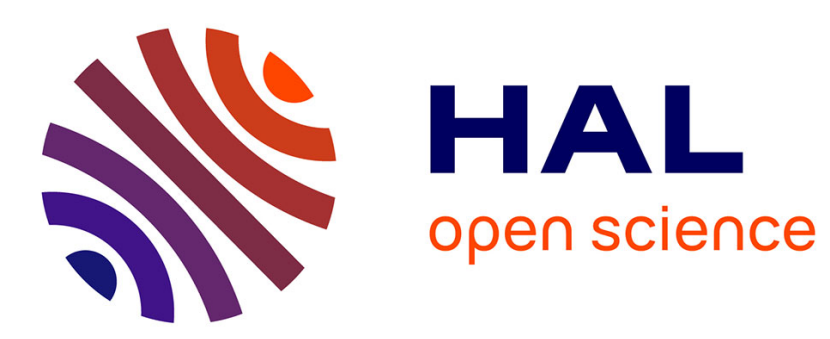

\title{
The degree ratio ranking method for directed graphs
}

René van den Brink, Agnieszka Rusinowska

\section{To cite this version:}

René van den Brink, Agnieszka Rusinowska. The degree ratio ranking method for directed graphs. European Journal of Operational Research, 2021, 288 (2), pp.563-575. 10.1016/j.ejor.2020.06.013 . hal-03153475

\section{HAL Id: hal-03153475 \\ https://hal.science/hal-03153475}

Submitted on 5 Jan 2022

HAL is a multi-disciplinary open access archive for the deposit and dissemination of scientific research documents, whether they are published or not. The documents may come from teaching and research institutions in France or abroad, or from public or private research centers.
L'archive ouverte pluridisciplinaire HAL, est destinée au dépôt et à la diffusion de documents scientifiques de niveau recherche, publiés ou non, émanant des établissements d'enseignement et de recherche français ou étrangers, des laboratoires publics ou privés. 


\title{
The Degree Ratio Ranking Method for Directed Graphs ${ }^{\text {tr }}$
}

\author{
René van den Brink ${ }^{1}$ \\ Department of Econometrics and Operations Research, and Tinbergen Institute, VU University \\ De Boelelaan 1105, 1081 HV Amsterdam, The Netherlands \\ Agnieszka Rusinowska \\ ${ }^{a}$ Paris School of Economics - CNRS, Université Paris 1, Centre d'Economie de la Sorbonne \\ 106-112 Bd de l'Hôpital, 75647 Paris Cedex 13, France
}

\begin{abstract}
One of the most famous ranking methods for digraphs is the ranking by Copeland score. The Copeland score of a node in a digraph is the difference between its outdegree (i.e. its number of outgoing arcs) and its indegree (i.e. its number of ingoing arcs). In the ranking by Copeland score, a node is ranked higher, the higher is its Copeland score. In this paper, we deal with an alternative method to rank nodes according to their out- and indegree, namely ranking the nodes according to their degree ratio, i.e. the outdegree divided by the indegree. To avoid dividing by zero, we add 1 to both the out- as well as indegree of every node. We provide an axiomatization of the ranking by degree ratio using a clone property, which says that the entrance of a clone or a copy (i.e. a node that is in some sense similar to the original node) does not change the ranking among the original nodes. We also provide a new axiomatization of the ranking by Copeland score using the same axioms except that this method satisfies a different clone property. Finally, we modify the ranking by degree ratio by taking only the out- and indegree, but by definition assume nodes with indegree zero to be ranked higher than nodes with positive indegree. We provide an axiomatization of this ranking method using yet another clone property and a maximal property. In this way, we can compare the three ranking methods by their clone property. Keywords: group decisions and negotiations, directed graph, ranking method, degree ratio, Copeland score
\end{abstract}

\footnotetext{
ฟh This research has been initiated when René van den Brink was Visiting Professor at the Centre d'Economie de la Sorbonne of the University of Paris 1. Agnieszka Rusinowska acknowledges the support by the National Agency for Research (Agence Nationale de la Recherche) - Project "CoCoRICo-CoDec" (ANR-14-CE24-0007-01). We thank three anonymous reviewers for their useful comments.

Email addresses: jrbrink@feweb.vu.nl (René van den Brink), agnieszka.rusinowska@univ-paris1.fr (Agnieszka Rusinowska)

${ }^{1}$ Corresponding author.
} 


\section{Introduction}

Ranking is one of the crucial issues in everyday economic, social, and political activities. There are numerous examples of applications, such as ranking departments and candidates within a university, journals ([17]), web pages on the internet ([7]), teams in sport competitions, firms in an industry, political candidates and alternatives in social choice ([19]). The use of an appropriate ranking method is of particular importance. Directed graphs have an established role in modeling and investigating various ranking methods, with nodes having different interpretations (individuals, journals, web pages, teams, alternatives, institutions, political parties, etc.).

Formally, a ranking method assigns to every directed graph a complete preorder on the set of nodes. One of the most famous ranking methods for digraphs is the ranking by Copeland score ([9]). The Copeland score of a node in a digraph is the difference between its outdegree (i.e. its number of outgoing arcs) and its indegree (i.e. its number of ingoing arcs). In the ranking by Copeland score, a node is ranked higher, the higher is its Copeland score. We investigate an alternative to rank nodes according to their out- and indegree, namely ranking the nodes according to their degree ratio, i.e. the outdegree divided by the indegree. To avoid dividing by a zero indegree, we add 1 to both the out- as well as indegree of every node. In some types of situations, the degree ratio ranking can give different results from the ranking by Copeland score. While a draw (i.e., two arcs between the same nodes oriented in opposite direction) is neutral in the ranking by Copeland score, it is not in our ranking by the degree ratio. In particular, when an agent loses more (respectively less) frequently than gains, a draw is beneficial (respectively disadvantageous) for such an agent. Therefore, when losing by itself is seen more negatively than winning is seen positively, the degree ratio ranking might be a more appropriate ranking method to use.

The degree ratio ranking method can be applied in various fields. In fact, it is applied in ranking players in online video gaming. When the nodes in a digraph represent countries (or regions) and the arcs represent migration flows, then the degree ratio gives for each country its emigration relative to (i.e. divided by) its immigration, which can be seen as an alternative to the usual net migration rate, being essentially a Copeland score. In case the digraph represents a financial network where outgoing arcs represent money borrowed to other banks, and ingoing arcs represent money borrowed from other banks, the degree ratio of a bank is its debit to credit ratio. A similar interpretation can be given when the digraph represents a mutual control network of firms investing in each other.

We provide an axiomatization of the ranking by degree ratio using a clone property, which says that the entrance of a copy or a clone of a node (i.e. a node that is similar to the original node) does not change the ranking between this node and the other original nodes. Other properties that, together with the clone property, characterize the ranking by degree ratio are anonymity, positive responsiveness, 
independence of irrelevant arcs, and the intermediary property. Anonymity means that relabeling the nodes implies a corresponding relabeling in the ranking. According to positive responsiveness, when one of two equally ranked nodes gets one more outgoing arc, this node becomes strictly higher ranked than the other one. Independence of irrelevant arcs implies that, when pairwise comparing two nodes, we only need to consider the arcs that involve these two nodes. The intermediary property means that putting only one new node on an existing arc, does not change the ranking.

Besides characterizing the ranking by degree ratio, we also provide a new axiomatization of the ranking by Copeland score using similar axioms, except that this ranking method satisfies a different clone property.

Finally, we modify the ranking by degree ratio by taking only the out- and indegree, but assuming that nodes with indegree zero are ranked higher than nodes with a positive indegree. Obviously, such a ranking by modified degree ratio is different from the ranking by degree ratio and the Copeland score. However, if the degree and modified degree ratios give different ranking of two nodes with positive indegrees, then their ranking by degree ratio is always the same as their ranking by Copeland score. We provide an axiomatic characterization of the ranking by modified degree ratio by similar axioms as mentioned before, except that we replace the clone property by yet another modified clone property and an additional maximal property. This last axiom requires to rank nodes with zero indegree above nodes with positive indegree. In this sense, the method refines the set of strong maximal nodes, being the set of nodes with indegree zero. If a strong maximal node exists, then the node that is ranked highest is a strong maximal node.

Related literature. There exists a vast literature that studies the Copeland score as well as other ranking methods. The ranking by Copeland score is characterized, e.g., by [15] and [3], see also [6] for a related ranking method. [18] characterizes the ranking by Copeland score on the class of tournaments. On the class of tournaments, the ranking by Copeland score coincides with the ranking by outdegree. In [22], a generalization of Rubinstein's result is presented by characterizing the ranking by outdegree for arbitrary directed graphs. An axiomatic characterization of the outflow ranking method for weighted directed graphs is provided in [23], while [21] axiomatizes the outflow as a relational power measure for weighted and nonweighted directed graphs. [5] axiomatizes the alternative $\beta$ ranking method for directed graphs, which is based on the $\beta$-measure introduced in [21]. Several works study methods based on evaluations or citations and consider one-sided or peers' settings; see e.g., [1], [16], [20]. In a one-sided setting, experts provide evaluations on some items to be ranked. We obtain the peers setting when the experts coincide with the items (e.g., webpages linking to other pages or journals citing each 
other). [14] studies ranking of items in a graph determined by a choice of utility function. [12] and [13] characterizes ranking methods based on evaluations or citations which consider one-sided settings and two-sided settings, respectively. The Hirsch index, which induces a ranking method supporting evaluations of researchers, is axiomatically characterized in [24] and [4]. For other works considering the peers setting, e.g., incentive compatibility, see [2], [8], and for some studies of rankings in a dynamic setting, see [10] and [11].

The underlying paper is structured as follows. In Section 2, we present basic preliminaries on digraphs and ranking methods. The ranking by degree ratio is introduced and axiomatized in Section 3 , In Section 4, we provide a comparable axiomatization of the ranking by Copeland score. The ranking by modified degree ratio is introduced and axiomatically characterized in Section 5 . We present some concluding remarks in Section 6 ,

\section{Preliminaries}

A directed graph (or digraph) is a pair $(N, D)$, where $N \subset \mathbb{N}$ is a finite set of nodes and $D \subset N \times N$ is a binary relation whose elements $(i, j) \in D$ are called $\operatorname{arcs}$ on $N$. We only consider digraphs $(N, D)$ that are irreflexive, i.e., $(i, i) \notin D$ for every $i \in N$. The collection of all digraphs is denoted by $\mathcal{D}$. For $i \in N$ and $(N, D) \in \mathcal{D}$, we define

$$
S_{(N, D)}(i)=\{j \in N \mid(i, j) \in D\}
$$

being the set of successors of node $i \in N$ in digraph $(N, D)$, and

$$
P_{(N, D)}(i)=\{j \in N \mid(j, i) \in D\}
$$

being the set of predecessors of $i$ in $(N, D)$. The outdegree out $(N, D)$ and indegree $i n_{i}(N, D)$ of node $i$ in $(N, D)$ are the cardinalities of $S_{(N, D)}(i)$ and $P_{(N, D)}(i)$, respectively, i.e.,

$$
\text { out }_{i}(N, D)=\# S_{(N, D)}(i) \text { and } i n_{i}(N, D)=\# P_{(N, D)}(i) .
$$

A preorder on $N$ is a binary relation $\mathcal{R} \subset N \times N$ that is reflexive (i.e., $(i, i) \in \mathcal{R}$ for all $i \in N$ ) and transitive (i.e., if $(i, j) \in \mathcal{R}$ and $(j, h) \in \mathcal{R}$, then $(i, h) \in \mathcal{R}$ for every $i, j, h \in N)$. A preorder $\mathcal{R}$ on $N$ is complete if $(i, j) \in \mathcal{R}$ or $(j, i) \in \mathcal{R}$ for every pair $i, j \in N, i \neq j$. We use the standard notation: $i \geq j$ if and only if $(i, j) \in \mathcal{R}$ (meaning that $i$ is ranked at least as high as $j$ ); $i>j$ if and only if $[i \geq j$ and not $j \geq i]$ (meaning that $i$ is ranked higher than $j$ ); and $i \sim j$ if and only if $[i \geq j$ as well as $j \geq i]$ (meaning that $i$ and $j$ are ranked equally). The collection of all complete preorders is denoted by $\mathcal{W}$. 
A ranking method is a mapping $R: \mathcal{D} \rightarrow \mathcal{W}$ which assigns to every digraph $(N, D) \in \mathcal{D}$ on $N$ a complete preorder $R(N, D) \in \mathcal{W}$ such that the set of nodes in $\mathcal{R}(N, D)$ coincides with the set of nodes in $(N, D)$. We use the notation $i \geq_{(N, D)} j$ if and only if $(i, j) \in R(N, D)$.

One of the most famous ranking methods for digraphs is the ranking by Copeland score. The Copeland score $\operatorname{cop}_{i}(N, D)$ of node $i \in N$ in digraph $(N, D)$ is given by

$$
\operatorname{cop}_{i}(N, D)=\operatorname{out}_{i}(N, D)-i n_{i}(N, D)
$$

Then, the ranking by Copeland score is the ranking method given by

$$
i \geq_{(N, D)}^{c o p} j \Leftrightarrow \operatorname{cop}_{i}(N, D) \geq \operatorname{cop}_{j}(N, D) \text { for all } i, j \in N .
$$

In the next section, we introduce and characterize a different ranking method based on the outdegree and indegree.

\section{Axiomatization of the ranking by degree ratio}

Whereas the Copeland score assigns to every node in a digraph its outdegree minus its indegree, the degree ratio assigns to every node in a digraph its outdegree divided by its indegree. To avoid dividing by zero, we add 1 to both the out- as well as indegree of every node. Notice that doing the same for the Copeland score does not change the scores, but it has an effect for the degree ratio.

Definition 1. The degree ratio of node $i \in N$ in digraph $(N, D)$ is given by

$$
r_{i}(N, D)=\frac{\text { out }_{i}(N, D)+1}{\operatorname{in}_{i}(N, D)+1} .
$$

The corresponding ranking method ranks nodes higher, the higher is their degree ratio.

Definition 2. The ranking by degree ratio is the ranking method given by

$$
i \geq_{(N, D)}^{d r} j \Leftrightarrow r_{i}(N, D) \geq r_{j}(N, D) \text { for all } i, j \in N .
$$

The ranking by degree ratio is different from the ranking by Copeland score, and it is easy to construct examples such that for two nodes $i, j \in N, \operatorname{cop}_{i}(N, D)<\operatorname{cop}_{j}(N, D)$ while $r_{i}(N, D)>r_{j}(N, D)$. This happens, e.g., when

$$
\operatorname{in}_{i}(N, D)=0 \quad \text { and } \quad \frac{\operatorname{out}_{j}(N, D)-i n_{j}(N, D)}{i n_{j}(N, D)+1}<\operatorname{out}_{i}(N, D)<\operatorname{out}_{j}(N, D)-i n_{j}(N, D) .
$$

Example 1. Consider the digraph $(N, D)$ with $N=\{1,2, \ldots, 9\}$ and $D=\{(1,2)\} \cup\{(i, 5) \mid i=3,4\} \cup$ $\{(5, i) \mid i=6,7,8,9\}$ as presented in Figure 1

Table 1 shows the out- and indegree, the Copeland score and the degree ratio for every node. The last row shows the modified degree ratio $\bar{r}$ which is discussed in Section 5

Note that $\operatorname{cop}_{1}(N, D)=1<2=\operatorname{cop}_{5}(N, D)$ but $r_{1}(N, D)=2>\frac{5}{3}=r_{5}(N, D)$. Moreover, $\frac{\text { out }_{5}(N, D)-i n_{5}(N, D)}{\operatorname{in}_{5}(N, D)+1}=\frac{2}{3}<1=$ out $_{1}(N, D)<2=\operatorname{out}_{5}(N, D)-\operatorname{in}_{5}(N, D)$. 


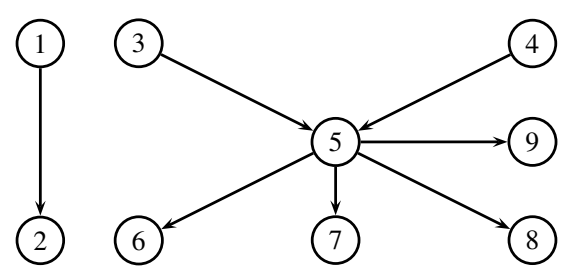

Figure 1: Example 1

\begin{tabular}{|c|c|c|c|}
\hline$i$ & $1,3,4$ & $2,6,7,8,9$ & 5 \\
\hline $\operatorname{out}_{i}(N, D)$ & 1 & 0 & 4 \\
\hline $\operatorname{in}_{i}(N, D)$ & 0 & 1 & 2 \\
\hline $\operatorname{cop}_{i}(N, D)$ & 1 & -1 & 2 \\
\hline$r_{i}(N, D)$ & 2 & $1 / 2$ & $5 / 3$ \\
\hline $\bar{r}_{i}(N, D)$ & $\infty$ & 0 & 2 \\
\hline
\end{tabular}

Table 1: Example1

Another property of the degree ratio concerns draws, i.e., two arcs between the same nodes oriented in opposite direction. A draw is not neutral in the degree ratio, contrary to the Copeland score which is indifferent to an additional draw. More precisely, when an agent loses more frequently than gains, he benefits from an additional draw. On the contrary, if an agent gains more frequently than loses, an additional draw is disadvantageous to him. So, one can say that being a winner requires keeping the good image. Formally, we have the following fact.

Fact 1. Let $(N, D),\left(N, D^{\prime}\right) \in \mathcal{D}, i \in N$ and $x \in \mathbb{N}_{+}$be such that $\operatorname{in}_{i}\left(N, D^{\prime}\right)=i n_{i}(N, D)+x$ and $\operatorname{out}_{i}\left(N, D^{\prime}\right)=\operatorname{out}_{i}(N, D)+x$. Then $\operatorname{cop}_{i}\left(N, D^{\prime}\right)=\operatorname{cop}_{i}(N, D)$ and

$$
r_{i}\left(N, D^{\prime}\right)>r_{i}(N, D) \quad \text { if and only if } \quad \operatorname{in}_{i}(N, D)>\text { out }_{i}(N, D) .
$$

ProOF

Indeed, we have

$$
\begin{gathered}
r_{i}\left(N, D^{\prime}\right)=\frac{\operatorname{out}_{i}\left(N, D^{\prime}\right)+1}{\operatorname{in}_{i}\left(N, D^{\prime}\right)+1}=\frac{\operatorname{out}_{i}(N, D)+x+1}{\operatorname{in}_{i}(N, D)+x+1}>\frac{\operatorname{out}_{i}(N, D)+1}{\operatorname{in}_{i}(N, D)+1}=r_{i}(N, D) \\
\left.\Leftrightarrow \operatorname{out}_{i}(N, D)+x+1\right)\left(\operatorname{in}_{i}(N, D)+1\right)>\left(\operatorname{out}_{i}(N, D)+1\right)\left(\operatorname{in}_{i}(N, D)+x+1\right) \\
\Leftrightarrow \operatorname{in}_{i}(N, D)>\operatorname{out}_{i}(N, D)
\end{gathered}
$$

Hence, with the out- and indegree being interpreted as the number of gains and losses, respectively, the degree ratio can be used, for instance, for ranking candidates in situations when losing is seen more negatively than winning is seen positively. 
In what follows, we deliver an axiomatic characterization of the ranking by degree ratio. The first axiom is the 'standard' anonymity axiom stating that relabeling the nodes implies a corresponding relabeling in the ranking. For digraph $(N, D) \in \mathcal{D}$ and permutation $\pi: N \rightarrow N$, the permuted digraph is $(N, \pi D)$ with $\pi D=\bigcup_{(i, j) \in D}(\pi(i), \pi(j))$.

Axiom 1 (Anonymity). For every $(N, D) \in \mathcal{D}$, it holds that $i \geq_{(N, D)} j \Leftrightarrow \pi(i) \geq_{(N, \pi D)} \pi(j)$.

Positive responsiveness implies that, when two nodes are equally ranked, and one of the nodes gets one more successor, then this node becomes strictly higher ranked than the other node.

Axiom 2 (Positive responsiveness). Let $(N, D),\left(N, D^{\prime}\right) \in \mathcal{D}$ and $i \in N$ be such that

$$
D^{\prime}=D \cup\{(i, g)\} \text { for some } g \in N \backslash\{i\} \text { such that }(i, g) \notin D \text {. }
$$

Then $i \geq_{(N, D)} j \Rightarrow i>_{\left(N, D^{\prime}\right)} j$ for all $j \in N \backslash\{i\}$.

The next axiom is independence of irrelevant arcs which implies that for the pairwise comparison between two nodes, we only need to consider the arcs that involve these two nodes.

Axiom 3 (Independence of irrelevant arcs). Let $(N, D),\left(N, D^{\prime}\right) \in \mathcal{D}$ and $i, j \in N$ be such that $S_{(N, D)}(i)=$ $S_{\left(N, D^{\prime}\right)}(i), P_{(N, D)}(i)=P_{\left(N, D^{\prime}\right)}(i), S_{(N, D)}(j)=S_{\left(N, D^{\prime}\right)}(j)$ and $P_{(N, D)}(j)=P_{\left(N, D^{\prime}\right)}(j)$. Then $i \geq_{(N, D)} j \Leftrightarrow$ $i \geq_{\left(N, D^{\prime}\right)} j$.

This axiom is weaker than independence of irrelevant arcs as used in [22], generalizing an axiom of [18], to axiomatize the ranking by outdegree. This requires that the ranking between two nodes does not change as long as their sets of successors do not change. An alternative weakening of [22]'s independence of irrelevant arcs is used by [5]. There an arc is considered irrelevant for the pairwise comparison between nodes $i$ and $j$, if none of these two nodes is predecessor on this arc, nor is any of their successors the successor on the arc. So, whereas we take into account the relations with the predecessors of $i$ and $j$, [5] takes account of the relations of the successors of $i$ and $j$ with their predecessors. These two weakenings of [22]'s independence of irrelevant arcs are not logically related.

All axioms above are also satisfied by the ranking by Copeland score. Anonymity, positive responsiveness and negative responsivenes 2 are used by [3] to axiomatize the ranking by Copeland score. Instead of independence of irrelevant arcs, there independence of 2- or 3- cycles is used, meaning that deleting a cycle of length 2 or 3 does not change the ranking.

\footnotetext{
${ }^{2}$ We do not need the axiom of negative responsiveness for characterizing the ranking by degree ratio, but in our framework this axiom would be defined as follows: Let $(N, D),\left(N, D^{\prime}\right) \in \mathcal{D}$ and $i \in N$ be such that $D^{\prime}=D \cup\{(g, i)\}$ for some $g \in N \backslash\{i\}$ such that $(g, i) \notin D$. Then $j \geq_{(N, D)} i \Rightarrow j>_{\left(N, D^{\prime}\right)} i$ for all $j \in N \backslash\{i\}$.
} 
Next, we introduce a new type of property that is not satisfied by the ranking by Copeland score. This concerns the entrance of new nodes. Suppose that node $i$ and all its direct relatives (i.e. all its successors and predecessors) get a 'clone' or a 'copy'. Assume that agent $i$ is symmetrically related to its own clones, i.e., it is its predecessor as well as successor. If agent $i$ is related to the copies of its relatives in exactly the same way as to its original relatives (i.e., $i$ becomes successor of the copies of all its predecessors, and becomes predecessor of the copies of all its successors), then the ranking between node $i$ and any other original node $j$ who is not related to node $i$, should not change.

Axiom 4 (Clone property 1). Let $(N, D) \in \mathcal{D}$ and $i \in N$, and let $S^{\prime}, P^{\prime} \subset \mathbb{N} \backslash N$ be such that $\# S^{\prime}=$ $\# S_{(N, D)}(i), \# P^{\prime}=\# P_{(N, D)}(i)$ and $\#\left(S^{\prime} \cap P^{\prime}\right)=\#\left(S_{(N, D)}(i) \cap P_{(N, D)}(i)\right)$. Further, let $\left(N^{\prime}, D^{\prime}\right)$ be given by $N^{\prime}=N \cup S^{\prime} \cup P^{\prime} \cup\left\{i^{\prime}\right\}, i^{\prime} \notin N \cup S^{\prime} \cup P^{\prime}$, and

$$
D^{\prime}=D \cup\left\{(h, i) \mid h \in P^{\prime}\right\} \cup\left\{(i, h) \mid h \in S^{\prime}\right\} \cup\left\{\left(i, i^{\prime}\right),\left(i^{\prime}, i\right)\right\}
$$

Then $i \geq_{(N, D)} j \Leftrightarrow i \geq_{\left(N^{\prime}, D^{\prime}\right)} j$ for all $j \in N \backslash\left(P_{(N, D)}(i) \cup S_{(N, D)}(i)\right)$.

The entrance of a clone is illustrated in Figure 2. (For transparency, the figure only considers cases where there are no nodes that are successor as well as predecessor of node $i$ ).

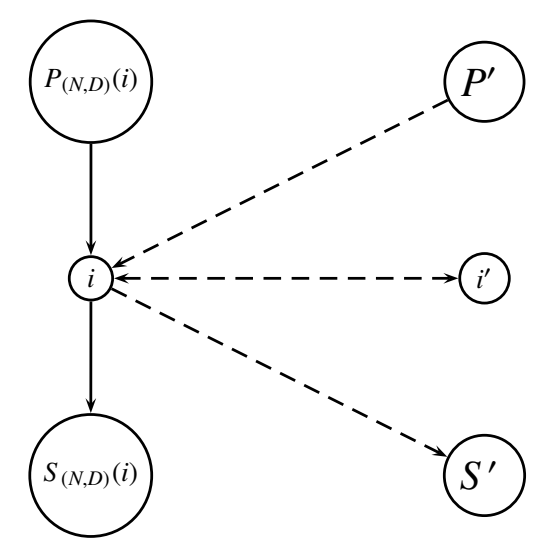

Figure 2: Axiom 4- entrance of a copy of $i$ if $P_{(N, D)}(i) \cap S_{(N, D)}(i)=\emptyset$, the dashed arrows represent the added arcs.

[5] considers a different axiom that allows new nodes to enter that are in some sense related to the existing nodes. First, they introduce node addition, which states that "the addition of nodes that are not linked to any other node has no influence on the ranking”. Obviously, this axiom is also satisfied by the ranking by degree ratio. Second, independence of local density is introduced, stating that "increasing the number of successors of node $i$ and simultaneously increasing their number of predecessors, in the same proportion, does not improve or worsen the position of node $i$ '. Together with node addition this is a different way of adding new nodes that are related to $i$ in a special way, but is logically not related to clone property 1 . 
Remark 1. In clone property 1 , we allowed symmetric arcs between the node that gets a clone or a copy and its successors/predecessors, i.e. $S_{(N, D)}(i) \cap P_{(N, D)}(i)$ might be nonempty. The uniqueness result presented below in Theorem $\square$ also holds if we weaken the clone property by not allowing symmetric arcs, i.e., if we only require the statement if $S_{(N, D)}(i) \cap P_{(N, D)}(i)=\emptyset$ for all $i \in N$, as was the case in the illustration in Figure 2

Remark 2. Also, we required the ranking not to change between $i$ and any original node $j$ that is not related to $i$. This is sufficient in the characterization, but the ranking by degree ratio satisfies the stronger axiom where we require the ranking between $i$ and any other node in $N$ not to change.

The final axiom states that putting only one new node between a successor and a predecessor, without any further relations, does not change the ranking. This axiom is also satisfied by the ranking by Copeland score.

Axiom 5 (Intermediary property). Let $(N, D) \in \mathcal{D}, z \notin N$ and $i, j \in N$ be such that $(i, j) \in D$. Let $\left(N^{\prime}, D^{\prime}\right)$ be given by $N^{\prime}=N \cup\{z\}$ and

$$
D^{\prime}=(D \backslash\{(i, j)\}) \cup\{(i, z),(z, j)\} .
$$

Then $h \geq_{(N, D)} g \Leftrightarrow h \geq_{\left(N^{\prime}, D^{\prime}\right)} g$ for all $h, g \in N$.

The above axioms characterize the ranking by degree ratio.

Theorem 1. The ranking by degree ratio is the unique ranking method satisfying anonymity, positive responsiveness, independence of irrelevant arcs, clone property 1, and the intermediary property.

We prove Theorem 1 in several steps.

Lemma 1. Let $(N, D) \in \mathcal{D}$ and $i, j \in N$ be such that $S_{(N, D)}(i) \cap P_{(N, D)}(i)=S_{(N, D)}(i) \cap P_{(N, D)}(j)=$ $S_{(N, D)}(j) \cap P_{(N, D)}(i)=S_{(N, D)}(j) \cap P_{(N, D)}(j)=\emptyset$, and $(i, j),(j, i) \notin D$. If ranking method $\geq$ satisfies the axioms of Theorem $\square$ then $\left[i \geq j\right.$ if and only if $\left.r_{i}(N, D) \geq r_{j}(N, D)\right]$.

PROOF

We distinguish the following cases:

Case A

Suppose that $r_{i}(N, D)=r_{j}(N, D)$. We will shortly denote $r_{i}(N, D)$ and $r_{j}(N, D)$ as $r_{i}$, respectively $r_{j}$.

First, we enter a cloning of node $i\left(i n_{j}(N, D)\right.$ times), and $j\left(i_{i}(N, D)\right.$ times) as in the clone property, in order to create symmetric positions for them.

To this end, define $P_{i}^{\prime}, P_{j}^{\prime}, S_{i}^{\prime}, S_{j}^{\prime}, I, J \subset \mathbb{N} \backslash N$ such that each pair of these sets is disjoint, and

- $\# I=i n_{j}(N, D), \# J=i n_{i}(N, D)$

- $\# P_{i}^{\prime}=\# P_{j}^{\prime}=i n_{i}(N, D) \cdot i n_{j}(N, D)$; 
- $\# S_{i}^{\prime}=\operatorname{out}_{i}(N, D) \cdot \operatorname{in}_{j}(N, D)$, and $\# S_{j}^{\prime}=\operatorname{out}_{j}(N, D) \cdot i n_{i}(N, D)$.

Further, let digraph $\left(N^{\prime}, D^{\prime}\right) \in \mathcal{D}$ be given by

$$
N^{\prime}=N \cup S_{i}^{\prime} \cup S_{j}^{\prime} \cup P_{i}^{\prime} \cup P_{j}^{\prime} \cup I \cup J
$$

and

$$
\begin{gathered}
D^{\prime}=D \cup\left(\bigcup_{h \in S_{i}^{\prime}}\{(i, h)\}\right) \cup\left(\bigcup_{h \in P_{i}^{\prime}}\{(h, i)\}\right) \cup\left(\bigcup_{h \in I}\{(i, h),(h, i)\}\right) \cup \\
\left(\bigcup_{h \in S_{j}^{\prime}}\{(j, h)\}\right) \cup\left(\bigcup_{h \in P_{j}^{\prime}}\{(h, j)\}\right) \cup\left(\bigcup_{h \in J}\{(j, h),(h, j)\}\right)
\end{gathered}
$$

This entrance of clones of nodes $i$ and $j$ is illustrated in Figure 3, where the dashed arrows represent new added arcs, and the solid arrows represent already existing $\operatorname{arcs}$ in $D$.

To get rid of the asymmetry between nodes $i$ and $j$ with respect to the different sizes of the sets $I$, respectively $J$, we put nodes on the arcs between $i$ (respectively $j$ ) and the nodes in the set $I$ (respectively the set $J$ ) in both directions, which does not influence the ranking between nodes $i$ and $j$ by the intermediary property. This gives the following digraph $\left(N^{\prime \prime}, D^{\prime \prime}\right)$. Without loss of generality, let the nodes in $I$ be labeled as $I=\left\{i_{1}, \ldots, i_{s}\right\}$, and thus $\# I=i n_{j}(N, D)=s$. Similarly, let the nodes in $J$ be labeled as $J=\left\{j_{1}, \ldots, j_{t}\right\}$, and thus \#J=inin $(N, D)=t$. Now, let $N^{\prime \prime}=N^{\prime} \cup I^{\prime} \cup J^{\prime}$ with $I^{\prime}, J^{\prime} \subset \mathbb{N} \backslash N^{\prime}$, $\# I^{\prime}=2 \# I$ and $\# J^{\prime}=2 \# J$, and suppose that the nodes in the two new sets are labeled as follows: $I^{\prime}=\left\{i_{11}^{\prime}, \ldots, i_{1 s}^{\prime}, i_{21}^{\prime}, \ldots, i_{2 s}^{\prime}\right\}$ and $J^{\prime}=\left\{j_{11}^{\prime}, \ldots, j_{1 t}^{\prime}, j_{21}^{\prime}, \ldots, j_{2 t}^{\prime}\right\}$. Further, let

$$
\begin{aligned}
& D^{\prime \prime}=\left(D^{\prime} \backslash\left(\left(\bigcup_{h \in I}\{(i, h),(h, i)\}\right) \cup\left(\bigcup_{h \in J}\{(j, h),(h, j)\}\right)\right)\right) \\
& \cup\left(\bigcup_{k=1}^{s}\left\{\left(i, i_{1 k}^{\prime}\right),\left(i_{1 k}^{\prime}, i_{k}\right),\left(i_{k}, i_{2 k}^{\prime}\right),\left(i_{2 k}^{\prime}, i\right)\right\}\right) \\
& \cup\left(\bigcup_{k=1}^{t}\left\{\left(j, j_{1 k}^{\prime}\right),\left(j_{1 k}^{\prime}, j_{k}\right),\left(j_{k}, j_{2 k}^{\prime}\right),\left(j_{2 k}^{\prime}, j\right)\right\}\right)
\end{aligned}
$$

being the digraph where we put intermediary nodes on all arcs between $i$, respectively $j$, and the nodes in the sets $I$, respectively $J$.

Next, let $\left(N^{\prime \prime}, D^{\prime \prime \prime}\right)$ be given by

$$
D^{\prime \prime \prime}=\left\{(h, g) \in D^{\prime \prime} \mid\{h, g\} \cap\{i, j\} \neq \emptyset\right\},
$$

where we deleted all 'irrelevant arcs' not containing $i$ or $j$. 


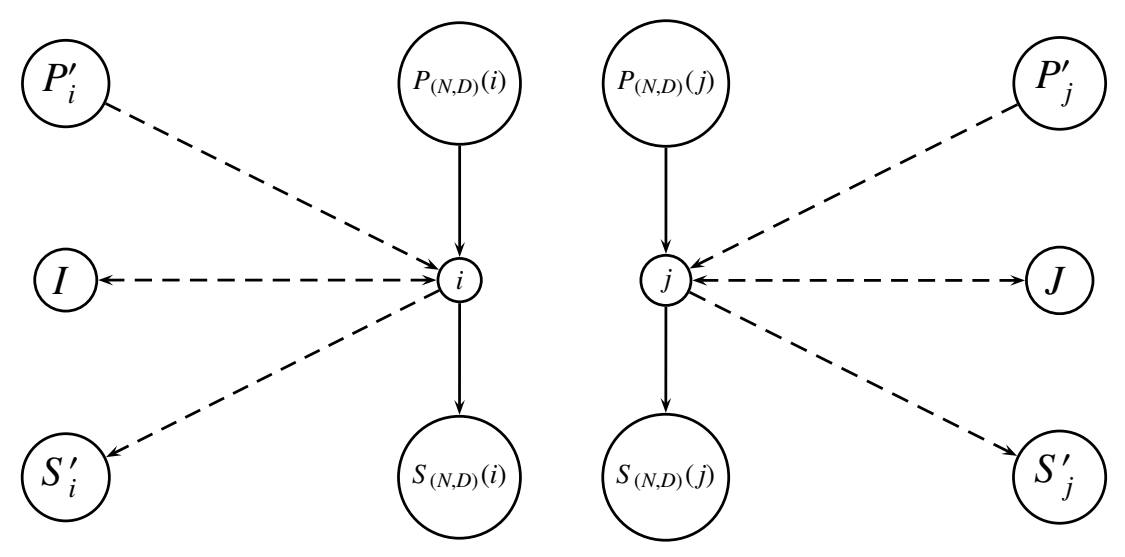

Figure 3: Proof of Lemma1- Case A

Notice that

(i) $\# P_{\left(N^{\prime \prime}, D^{\prime \prime \prime}\right)}(i)=\# P_{i}^{\prime}+\# P_{i}(N, D)+\# I=i n_{i}(N, D) \cdot i n_{j}(N, D)+i n_{i}(N, D)+i n_{j}(N, D)=\# P_{j}^{\prime}+\# J+$ $\# P_{j}(N, D)=\# P_{\left(N^{\prime \prime}, D^{\prime \prime \prime}\right)}(j)$, and

(ii) $\# S_{\left(N^{\prime \prime}, D^{\prime \prime \prime}\right)}(i)=\# S_{i}^{\prime}+\# S_{i}(N, D)+\# I=\operatorname{out}_{i}(N, D) \cdot i n_{j}(N, D)+\operatorname{out}_{i}(N, D)+i n_{j}(N, D)=\operatorname{out}_{i}(N, D)+$ 1) $\left(\operatorname{in}_{j}(N, D)+1\right)-1=\left(\operatorname{out}_{j}(N, D)+1\right)\left(\operatorname{in}_{i}(N, D)+1\right)-1=\operatorname{out}_{j}(N, D) \cdot \operatorname{in}_{i}(N, D)+$ out $_{j}(N, D)+\operatorname{in}_{i}(N, D)=$ $\# S_{j}^{\prime}+\# S_{j}(N, D)+\# J=\# S_{\left(N^{\prime \prime}, D^{\prime \prime \prime}\right)}(j)$, where the fourth equality follows from the assumption that $r_{i}(N, D)=r_{j}(N, D)$.

Therefore, anonymity implies that $i \sim_{\left(N^{\prime \prime}, D^{\prime \prime \prime}\right)} j$.

Independence of irrelevant arcs implies that the ranking between $i$ and $j$ is the same in $\left(N^{\prime \prime}, D^{\prime \prime}\right)$ as in $\left(N^{\prime \prime}, D^{\prime \prime \prime}\right)$, and thus $i \sim\left(N^{\prime \prime}, D^{\prime \prime}\right) j$.

The intermediary property implies that the ranking between $i$ and $j$ is the same in $\left(N^{\prime}, D^{\prime}\right)$ as in $\left(N^{\prime \prime}, D^{\prime \prime}\right)$, and thus $i \sim{ }_{\left(N^{\prime}, D^{\prime}\right)} j$.

A repeated application of clone property 1 implies that $i \sim_{(N, D)} j$.

\section{Case B}

Suppose without loss of generality that $r_{i}(N, D)>r_{j}(N, D)$.

We define similar sets $S_{i}^{\prime}, S_{j}^{\prime}, P_{i}^{\prime}, P_{j}^{\prime}, I$ and $J$ as in Case A, so define $P_{i}^{\prime}, P_{j}^{\prime}, S_{i}^{\prime}, S_{j}^{\prime}, I, J \subset \mathbb{N} \backslash N$ such that each pair of these sets is disjoint, and

- $\# I=i n_{j}(N, D), \# J=i n_{i}(N, D)$

- $\# P_{i}^{\prime}=\# P_{j}^{\prime}=i n_{i}(N, D) \cdot i n_{j}(N, D)$

- $\# S_{i}^{\prime}=\operatorname{out}_{i}(N, D) \cdot i n_{j}(N, D)$, and $\# S_{j}^{\prime}=\operatorname{out}_{j}(N, D) \cdot i n_{i}(N, D)$. 
Define digraph $\left(N^{\prime}, D^{\prime}\right)$ as in Case A (see (2) and (3)).

Similar to Case A, we put intermediary nodes on the arcs between nodes $i$ (respectively $j$ ) and the nodes in the set $I$ (respectively the set $J$ ), to obtain the digraph $\left(N^{\prime \prime}, D^{\prime \prime}\right)$ in the same way as in Case A, see (4). Also, similar to Case A, let $\left(N^{\prime \prime}, D^{\prime \prime \prime}\right)$ be the digraph where we deleted the 'irrelevant arcs', given by (5).

Similar to Case A,

(i) $\# P_{\left(N^{\prime \prime}, D^{\prime \prime \prime}\right)}(i)=\# P_{i}^{\prime}+\# P_{i}(N, D)+\# I=i n_{i}(N, D) \cdot i n_{j}(N, D)+i n_{i}(N, D)+i n_{j}(N, D)=\# P_{j}^{\prime}+\# J+$ $\# P_{j}(N, D)=\# P_{\left(N^{\prime \prime}, D^{\prime \prime \prime}\right)}(j)$.

But now,

(ii) $\# S_{\left(N^{\prime \prime}, D^{\prime \prime \prime}\right)}(i)=\# S_{i}^{\prime}+\# S_{i}(N, D)+\# I=\operatorname{out}_{i}(N, D) \cdot i n_{j}(N, D)+\operatorname{out}_{i}(N, D)+i n_{j}(N, D)=\left(\operatorname{out}_{i}(N, D)+\right.$ 1) $\left(\operatorname{in}_{j}(N, D)+1\right)-1>\left(\operatorname{out}_{j}(N, D)+1\right)\left(\operatorname{in}_{i}(N, D)+1\right)-1=\operatorname{out}_{j}(N, D) \cdot \operatorname{in}_{i}(N, D)+$ out $_{j}(N, D)+$ $i n_{i}(N, D)=\# S_{j}^{\prime}+\# S_{j}(N, D)+\# J=\# S_{\left(N^{\prime \prime}, D^{\prime \prime \prime}\right)}(j)$, where the inequality follows from the assumption that $r_{i}(N, D)>r_{j}(N, D)$.

Let $D^{\prime \prime \prime \prime} \subset D^{\prime \prime \prime}$ with $\left[(h, g) \in D^{\prime \prime \prime} \backslash D^{\prime \prime \prime \prime} \Rightarrow h=i\right]$ and $\# S_{\left(N^{\prime \prime}, D^{\prime \prime \prime}\right)}(i)=\# S_{\left(N^{\prime \prime}, D^{\prime \prime \prime}\right)}(j)=\# S_{\left(N^{\prime \prime}, D^{\prime \prime \prime}\right)}(j)$.

Since $\left(N^{\prime \prime}, D^{\prime \prime \prime \prime}\right)$ is as in Case A, we have that $i \sim_{\left(N^{\prime \prime}, D^{\prime \prime \prime \prime}\right)} j$.

Since $D^{\prime \prime \prime}$ is obtained from $D^{\prime \prime \prime \prime}$ by adding arcs with node $i$ as predecessor, a repeated application of positive responsiveness implies that $i>_{\left(N^{\prime \prime}, D^{\prime \prime \prime}\right)} j$.

Independence of irrelevant arcs implies that the ranking between $i$ and $j$ is the same in $\left(N^{\prime \prime}, D^{\prime \prime}\right)$ as in $\left(N^{\prime \prime}, D^{\prime \prime \prime}\right)$, and thus $i>_{\left(N^{\prime \prime}, D^{\prime \prime}\right)} j$.

The intermediary property implies that the ranking between $i$ and $j$ is the same in $\left(N^{\prime}, D^{\prime}\right)$ as in $\left(N^{\prime \prime}, D^{\prime \prime}\right)$, and thus $i>_{\left(N^{\prime}, D^{\prime}\right)} j$.

A repeated application of clone property 1 implies that $i \geq_{(N, D)} j$.

In a similar way, it can be shown that $j \nsucceq_{(N, D)} i$, and thus $i>_{(N, D)} j$. 


\section{Proof of Theorem 1}

To prove uniqueness, given Lemma 1, we still have to consider the case where not all conditions $S_{(N, D)}(i) \cap P_{(N, D)}(i)=S_{(N, D)}(i) \cap P_{(N, D)}(j)=S_{(N, D)}(j) \cap P_{(N, D)}(i)=S_{(N, D)}(j) \cap P_{(N, D)}(j)=\emptyset$ and $(i, j),(j, i) \notin D$ are satisfied. This is dealt with by the intermediary property.

First, if $(i, j) \in D$ then define $\left(N^{\prime}, D^{\prime}\right)$ by $N^{\prime}=N \cup\{z\}$ for some $z \notin N$, and $D^{\prime}=(D \backslash\{(i, j)\}) \cup\{(i, z),(z, j)\}$. Similarly if $(j, i) \in D$. If $(i, j),(j, i) \notin D$, then simply take $\left(N^{\prime}, D^{\prime}\right)=(N, D)$.

Next, define

$$
\begin{gathered}
H=\left(\bigcup_{h \in S_{(N, D)}(i) \cap P_{(N, D)}(i)}\{(i, h)\}\right) \cup\left(\bigcup_{h \in S_{(N, D)}(i) \cap P_{(N, D)}(j)}\{(i, h)\}\right) \cup \\
\left(\bigcup_{h \in P_{(N, D)}(i) \cap S_{(N, D)}(j)}\{(j, h)\}\right) \cup\left(\bigcup_{h \in S_{(N, D)}(j) \cap P_{(N, D)}(j)}\{(j, h)\}\right),
\end{gathered}
$$

being the outgoing arcs (from $i$ or $j$ ) of the symmetric part involving nodes $i$ and $j$, and nodes that are directly related to both $i$ and $j$.

Then, define $\left(N^{\prime \prime}, D^{\prime \prime}\right)$ such that

$$
N^{\prime} \subset N^{\prime \prime} \text { with } \# N^{\prime \prime}=\# N^{\prime}+\# H
$$

and, labeling the nodes in $N^{\prime \prime} \backslash N^{\prime}$ as $N^{\prime \prime} \backslash N^{\prime}=\left\{z_{(h, g)} \mid(h, g) \in H\right\}$,

$$
D^{\prime \prime}=\left(D^{\prime} \backslash H\right) \cup\left\{\bigcup_{(h, g) \in H}\left\{\left(h, z_{(h, g)}\right),\left(z_{(h, g)}, g\right)\right\}\right\} .
$$

Thus, $\operatorname{in}_{z}\left(N^{\prime \prime}, D^{\prime \prime}\right)=\operatorname{out}_{z}\left(N^{\prime \prime}, D^{\prime \prime}\right)=1$ for all $z \in N^{\prime \prime} \backslash N^{\prime}$.

Since $\left(N^{\prime \prime}, D^{\prime \prime}\right)$ is as in Lemma1, we have $i \geq_{\left(N^{\prime \prime}, D^{\prime \prime}\right)} j$ if and only if $i \geq_{\left(N^{\prime \prime}, D^{\prime \prime}\right)}^{d r} j$.

The intermediary property then implies that $i \geq_{(N, D)} j$ if and only if $i \geq_{(N, D)}^{d r} j$.

We are left to show that the ranking by degree ratio satisfies the axioms.

$\geq^{d r}$ satisfying anonymity is obvious.

$\geq^{d r}$ satisfying positive responsiveness follows since the degree ratio of a node is increasing if its outdegree increases, which has no positive effect for the other nodes.

$z^{d r}$ satisfying independence of irrelevant arcs follows since the degree ratio of a node only depends on the arcs with its successors and predecessors.

$\geq^{d r}$ satisfying clone property 1 follows since $r_{i}\left(N^{\prime}, D^{\prime}\right)=\frac{o u t_{i}\left(N^{\prime}, D^{\prime}\right)+1}{i i_{i}\left(N^{\prime}, D^{\prime}\right)+1}=\frac{2 o u t_{i}(N, D)+1+1}{2 i i_{i}(N, D)+1+1}=\frac{2\left(o u t_{i}(N, D)+1\right)}{2\left(i_{i}(N, D)+1\right)}=$ $\frac{\text { out }_{i}(N, D)+1}{i i_{i}(N, D)+1}=r_{i}(N, D)$, and $r_{j}\left(N^{\prime}, D^{\prime}\right)=r_{j}(N, D)$ for all $j \in N \backslash\{i\}$, where $\left(N^{\prime}, D^{\prime}\right)$ is as given in the axiom.

$\geq^{d r}$ satisfying the intermediary property follows since $r_{j}\left(N^{\prime}, D^{\prime}\right)=r_{j}(N, D)$ for all $j \in N$ with $\left(N^{\prime}, D^{\prime}\right)$ as given in the axiom. 
Logical independence of the conditions in Theorem 1 is shown by the following alternative ranking methods.

1. Consider the ranking method that always ranks all nodes according to their label: For every $(N, D) \in \mathcal{D}$, let

$$
i>_{(N, D)} j \Leftrightarrow i<j \text { for all } i, j \in N \text {. }
$$

This ranking method satisfies all axioms of Theorem 1 except anonymity.

2. Consider the ranking method that always ranks all nodes equally irrespective of the relations among the nodes: For every $(N, D) \in \mathcal{D}$, let

$$
i \sim_{(N, D)} j \text { for all } i, j \in N \text {. }
$$

This ranking method satisfies all axioms of Theorem 1 except positive responsiveness.

3. Consider the ranking method, where (i) node $i$ is ranked higher than node $j$ if there is a directed path from $i$ to $j$ but not the other way around, or (ii) if there is a directed path in both directions or no directed path between these nodes at all, then they are ranked by their degree ratio. The transitive closure of digraph $(N, D)$ is digraph $(N, \operatorname{tr}(D))$ given by $(i, j) \in \operatorname{tr}(D)$ if and only if there exists a sequence $h_{1}, \ldots, h_{t} \in N$ such that (i) $h_{1}=i$, (ii) $\left(h_{k}, h_{k+1}\right) \in D$ for all $k \in\{1, \ldots, t-1\}$, and (iii) $h_{t}=j$. For every $(N, D) \in \mathcal{D}$, let

$$
i \geq_{(N, D)} j \Leftrightarrow\left\{\begin{array}{l}
(i, j) \in \operatorname{tr}(D) \text { and }(j, i) \notin \operatorname{tr}(D) \\
\text { or } \\
(i, j),(j, i) \in \operatorname{tr}(D) \text { and } r_{i}(N, D) \geq r_{j}(N, D) \\
\text { or } \\
(i, j),(j, i) \notin \operatorname{tr}(D) \text { and } r_{i}(N, D) \geq r_{j}(N, D)
\end{array}\right.
$$

This ranking method satisfies all axioms of Theorem 1 except independence of irrelevant arcs.

4. The ranking by Copeland score satisfies all axioms of Theorem 1 except clone property 1 (see Section 4).

5. Consider the modification of the ranking by degree ratio where the outdegree (respectively indegree) is modified by assigning only $\frac{1}{2}$ to every successor that is also a predecessor (respectively assigning only $\frac{1}{2}$ to every predecessor that is also a successor). Define

$$
\widehat{r}_{i}(N, D)=\frac{\#\left(S_{(N, D)}(i) \backslash P_{(N, D)}(i)\right)+\frac{1}{2} \#\left(S_{(N, D)}(i) \cap P_{(N, D)}(i)\right)+\frac{1}{2}}{\#\left(P_{(N, D)}(i) \backslash S_{(N, D)}(i)\right)+\frac{1}{2} \#\left(S_{(N, D)}(i) \cap P_{(N, D)}(i)\right)+\frac{1}{2}}
$$


For every $(N, D) \in \mathcal{D}$, let

$$
i \geq j \Leftrightarrow \widehat{r}_{i}(N, D) \geq \widehat{r}_{j}(N, D)
$$

This ranking method satisfies all axioms of Theorem 1 except the intermediary property.

\section{A comparable axiomatization of the ranking by Copeland score}

From the axioms of Theorem 1 , the ranking by Copeland score satisfies all except the clone property. However, it does satisfy an alternative clone property where, instead of putting all arcs as described in Axiom 4, we only link $i$ with its own copy $i^{\prime}$ by putting the arcs between $i$ and its copy $i^{\prime}$ in both directions. So, we only consider cloning $i^{\prime}$ as new entrant, who is symmetrically related to $i$. This entrance of a clone is illustrated in Figure 4 (For transparency, the figure again only considers cases where there are no nodes that are successors as well as predecessors of node $i$ ).

Axiom 6 (Clone property 2). Let $(N, D) \in \mathcal{D}$ and $i \in N$. Let $\left(N^{\prime}, D^{\prime}\right)$ be given by $N^{\prime}=N \cup\left\{i^{\prime}\right\}, i^{\prime} \notin N$, and $D^{\prime}=D \cup\left\{\left(i, i^{\prime}\right),\left(i^{\prime}, i\right)\right\}$. Then $i \geq_{(N, D)} j \Leftrightarrow i \geq_{\left(N^{\prime}, D^{\prime}\right)} j$ for all $j \in N$.

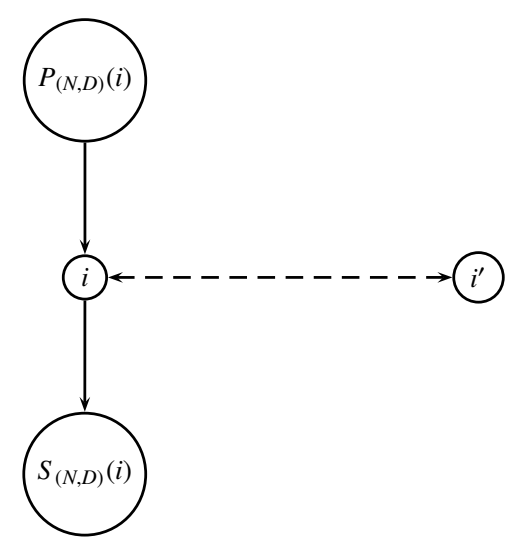

Figure 4: Axiom 6- entrance of a copy of $i$ if $P_{(N, D)}(i) \cap S_{(N, D)}(i)=\emptyset$, the dashed arrows represent the added arcs.

Replacing clone property 1 in Theorem 1 by clone property 2 , characterizes the ranking by Copeland score.

Theorem 2. The ranking by Copeland score is the unique ranking method satisfying anonymity, positive responsiveness, independence of irrelevant arcs, clone property 2, and the intermediary property.

We prove Theorem 2 in similar steps as in the proof of Theorem 1 .

Lemma 2. Let $(N, D) \in \mathcal{D}$ and $i, j \in N$ be such that $S_{(N, D)}(i) \cap P_{(N, D)}(i)=S_{(N, D)}(i) \cap P_{(N, D)}(j)=$ $S_{(N, D)}(j) \cap P_{(N, D)}(i)=S_{(N, D)}(j) \cap P_{(N, D)}(j)=\emptyset$, and $(i, j),(j, i) \notin D$. If ranking method $\geq$ satisfies the axioms of Theorem 2 then $\left[i \geq j\right.$ if and only if $\left.\operatorname{cop}_{i}(N, D) \geq \operatorname{cop}_{j}(N, D)\right]$. 
Proof

We distinguish two similar cases as in the proof of Theorem 1

Case A

Suppose that $\operatorname{cop}_{i}(N, D)=\operatorname{cop}_{j}(N, D)$. Suppose without loss of generality that $\operatorname{out}_{i}(N, D) \geq \operatorname{out}_{j}(N, D)$ (and thus $\operatorname{in}_{i}(N, D) \geq i n_{j}(N, D)$ ).

First, we enter a cloning of node $j$ several times as in clone property 2 , in order to create symmetric positions for nodes $i$ and $j$. (Notice that in the proof of Theorem 1 we had to enter copies of both nodes $i$ and $j$.) Therefore, for $J \subset \mathbb{N} \backslash N$ such that $\# J=\operatorname{out}_{i}(N, D)-\operatorname{out}_{j}(N, D)$, define digraph $\left(N^{\prime}, D^{\prime}\right) \in \mathcal{D}$ by

$$
N^{\prime}=N \cup J
$$

and

$$
D^{\prime}=D \cup\left(\bigcup_{h \in J}\{(h, j),(j, h)\}\right) .
$$

The entrance of a cloning of node $j$ is illustrated in Figure 5, where the dashed arrows represent new added arcs, and the solid arrows represent already existing arcs.

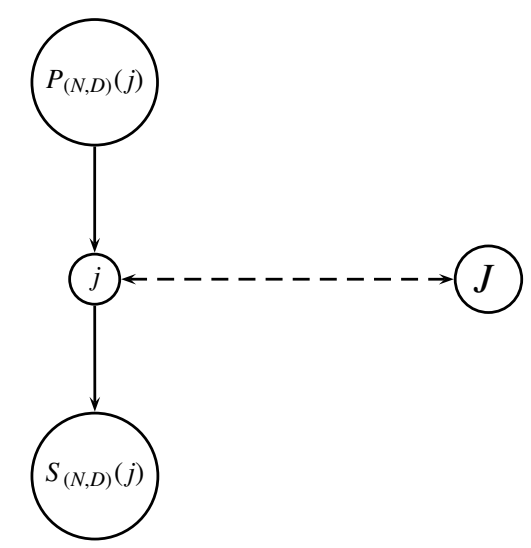

Figure 5: Proof of Lemma2- Case A

To get rid of the asymmetry between nodes $i$ and $j$ with respect to the set $J$, we add intermediary nodes between $j$ and the nodes in the set $J$, which does not influence the ranking between nodes $i$ and $j$ by the intermediary property. This is done similar as in the proof of Theorem 1, but now only considering $j$ and the set $J$. This gives the following digraph $\left(N^{\prime \prime}, D^{\prime \prime}\right)$. Without loss of generality, let the nodes in $J$ be labeled as $J=\left\{j_{1}, \ldots, j_{t}\right\}$ and thus \#J=out $(N, D)-\operatorname{out}_{j}(N, D)=t$. Now, let $N^{\prime \prime}=N^{\prime} \cup J^{\prime}$ with $J^{\prime} \subset \mathbb{N} \backslash N^{\prime}$ such that $\# J^{\prime}=2 \# J$, and suppose that the nodes in the new set are labeled as follows: 
$J^{\prime}=\left\{j_{11}^{\prime}, \ldots, j_{1 t}^{\prime}, j_{21}^{\prime}, \ldots, j_{2 t}^{\prime}\right\}$. Further, let

$$
\begin{gathered}
D^{\prime \prime}=\left(D^{\prime} \backslash\left(\bigcup_{h \in J}\{(j, h),(h, j)\}\right)\right) \\
\cup\left(\bigcup_{k=1}^{t}\left\{\left(j, j_{1 k}^{\prime}\right),\left(j_{1 k}^{\prime}, j_{k}\right),\left(j_{k}, j_{2 k}^{\prime}\right),\left(j_{2 k}^{\prime}, j\right)\right\}\right)
\end{gathered}
$$

being the digraph where we put intermediary nodes on all arcs between $j$ and the nodes in the set $J$.

Similar to the proof of Theorem 1, let $\left(N^{\prime \prime}, D^{\prime \prime \prime}\right)$ be given by

$$
D^{\prime \prime \prime}=\left\{(h, g) \in D^{\prime \prime} \mid\{h, g\} \cap\{i, j\} \neq \emptyset\right\},
$$

where we deleted all 'irrelevant arcs' not containing $i$ or $j$.

Notice that $\# S_{\left(N^{\prime \prime}, D^{\prime \prime \prime}\right)}(j)=\# S_{(N, D)}(j)+\# J=\operatorname{out}_{j}(N, D)+o u t_{i}(N, D)-o u t_{j}(N, D)=\operatorname{out}_{i}(N, D)=$ $\# S_{\left(N^{\prime \prime}, D^{\prime \prime \prime}\right)}(i)$, and thus with $\operatorname{cop}_{i}(N, D)=\operatorname{cop}_{j}(N, D)$ it follows that $\# P_{\left(N^{\prime \prime}, D^{\prime \prime \prime}\right)}(j)=\# P_{(N, D)}(j)+\# J=$ $\operatorname{in}_{j}(N, D)+\operatorname{out}_{i}(N, D)-$ out $_{j}(N, D)=i n_{i}(N, D)=\# P_{\left(N^{\prime \prime}, D^{\prime \prime \prime}\right)}(i)$.

Therefore, anonymity implies that $i \sim_{\left(N^{\prime \prime}, D^{\prime \prime \prime}\right)} j$.

Independence of irrelevant arcs implies that the ranking between $i$ and $j$ is the same in $\left(N^{\prime \prime}, D^{\prime \prime}\right)$ as in $\left(N^{\prime \prime}, D^{\prime \prime \prime}\right)$, and thus $i \sim_{\left(N^{\prime \prime}, D^{\prime \prime}\right)} j$.

The intermediary property implies that the ranking between $i$ and $j$ is the same in $\left(N^{\prime}, D^{\prime}\right)$ as in $\left(N^{\prime \prime}, D^{\prime \prime}\right)$, and thus $i \sim_{\left(N^{\prime}, D^{\prime}\right)} j$.

Similar to the proof of Theorem 1 a repeated application of clone property 2 implies that $i \sim_{(N, D)} j$.

\section{Case B}

Suppose without loss of generality that $\operatorname{cop}_{i}(N, D)>\operatorname{cop}_{j}(N, D)$.

If $i n_{i}(N, D)<i n_{j}(N, D)$ then take $I \subset \mathbb{N} \backslash N$ with $\# I=i n_{j}(N, D)-i n_{i}(N, D)$, and let $\left(N^{\prime}, D^{\prime}\right) \in \mathcal{D}$ be given by $N^{\prime}=N \cup I$ and $D^{\prime}=D \cup\left(\bigcup_{h \in I}\{(i, h),(h, i)\}\right)$. Since $i n_{i}\left(N^{\prime}, D^{\prime}\right)=i n_{j}\left(N^{\prime}, D^{\prime}\right)$ and $\operatorname{cop}_{i}\left(N^{\prime}, D^{\prime}\right)=$ $\operatorname{cop}_{i}(N, D)>\operatorname{cop}_{j}(N, D)=\operatorname{cop}_{j}\left(N^{\prime}, D^{\prime}\right)$, it follows that $\operatorname{out}_{i}\left(N^{\prime}, D^{\prime}\right)>\operatorname{out}_{j}\left(N^{\prime}, D^{\prime}\right)$.

Suppose that the nodes in $I$ are labeled as $I=\left\{i_{1}, \ldots, i_{p}\right\}$, and thus $p=\# I=i n_{j}(N, D)-i n_{i}(N, D)$. Further, let $I^{\prime} \subset \mathbb{N} \backslash N^{\prime}$ with $\# I^{\prime}=2 \# I$, and suppose that the nodes in $I^{\prime}$ are labeled as $I^{\prime}=\left\{i_{11}, \ldots, i_{1 p}, i_{21}, \ldots i_{2 p}\right\}$. Define $\left(N^{\prime \prime}, D^{\prime \prime}\right)$ by $N^{\prime \prime}=N^{\prime} \cup I^{\prime}$ and

$$
\left.D^{\prime \prime}=\left(D^{\prime} \backslash\left(\bigcup_{h \in I}(i, h),(h, i)\right\}\right)\right) \cup\left(\bigcup_{k=1}^{p}\left\{\left(i, i_{1 k}^{\prime}\right),\left(i_{k 1}^{\prime}, i_{k}\right),\left(i_{k}, i_{2 k}^{\prime}\right),\left(i_{2 k}^{\prime}, i\right)\right\}\right),
$$

being the directed graph where we put intermediary nodes on symmetric arcs between $i$ and the nodes in the set $I$, in both directions. 
Notice that $\operatorname{in}_{i}\left(N^{\prime \prime}, D^{\prime \prime}\right)=\operatorname{in}_{j}\left(N^{\prime \prime}, D^{\prime \prime}\right)=\operatorname{in}_{j}(N, D)$ and $\operatorname{out}_{i}\left(N^{\prime \prime}, D^{\prime \prime}\right)=\operatorname{out}_{i}(N, D)+\# I=\operatorname{out}_{i}(N, D)+$ $\operatorname{in}_{j}(N, D)-\operatorname{in}_{i}(N, D)>\operatorname{out}_{j}(N, D)=\operatorname{out}_{j}\left(N^{\prime \prime}, D^{\prime \prime}\right)$, where the inequality follows from $\operatorname{cop}_{i}(N, D)>$ $\operatorname{cop}_{j}(N, D)$.

Let $\left(N^{\prime \prime}, D^{\prime \prime \prime}\right) \in \mathcal{D}$ be such that $D^{\prime \prime \prime} \subset D^{\prime \prime}$ with

- $(h, g) \in D^{\prime \prime} \backslash D^{\prime \prime \prime} \Rightarrow h=i$, and

- $\operatorname{out}_{i}\left(N^{\prime \prime}, D^{\prime \prime \prime}\right)=\operatorname{out}_{j}\left(N^{\prime \prime}, D^{\prime \prime \prime}\right)=\operatorname{out}_{j}\left(N^{\prime \prime}, D^{\prime \prime}\right)$.

Since $\left(N^{\prime \prime}, D^{\prime \prime \prime}\right)$ is as in Case A, we have that $i \sim_{\left(N^{\prime \prime}, D^{\prime \prime \prime}\right)} j$.

Since $D^{\prime \prime}$ is obtained from $D^{\prime \prime \prime}$ by adding arcs with node $i$ as predecessor, a repeated application of positive responsiveness implies that $i>_{\left(N^{\prime \prime}, D^{\prime \prime}\right)} j$.

The intermediary property implies that the ranking between $i$ and $j$ is the same in $\left(N^{\prime}, D^{\prime}\right)$ as in $\left(N^{\prime \prime}, D^{\prime \prime}\right)$, and thus $i>_{\left(N^{\prime}, D^{\prime}\right)} j$.

A repeated application of clone property 2 implies that $i \geq_{(N, D)} j$.

In a similar way, it can be shown that $j \nsucceq_{(N, D)} i$, and thus $i>_{(N, D)} j$.

If $i n_{i}(N, D)>i n_{j}(N, D)$, we start by taking $J \subset \mathbb{N} \backslash N$ with $\# J=i n_{i}(N, D)-i n_{j}(N, D)$, and let $\left(N^{\prime}, D^{\prime}\right) \in \mathcal{D}$ be given by $N^{\prime}=N \cup J$ and $D^{\prime}=D \cup\left(\bigcup_{h \in J}\{(j, h),(h, j)\}\right.$ ). (Again, $i n_{i}\left(N^{\prime}, D^{\prime}\right)=i n_{j}\left(N^{\prime}, D^{\prime}\right)$ and $\operatorname{out}_{i}\left(N^{\prime}, D^{\prime}\right)>\operatorname{out}_{j}\left(N^{\prime}, D^{\prime}\right)$.) Then, the proof follows a similar reasoning as above, but now replacing $\left(N^{\prime \prime}, D^{\prime \prime}\right)$ by the similar digraph that is obtained by putting intermediary nodes on symmetric arcs between $j$ and the nodes in the set $J$, in both directions, and defining $D^{\prime \prime \prime}$ in the same way as above.

Proof of THeOREM 2

To prove uniqueness, given Lemma 2, we still have to consider the case where not all conditions $S_{(N, D)}(i) \cap P_{(N, D)}(i)=S_{(N, D)}(i) \cap P_{(N, D)}(j)=S_{(N, D)}(j) \cap P_{(N, D)}(i)=S_{(N, D)}(j) \cap P_{(N, D)}(j)=\emptyset$ and $(i, j),(j, i) \notin D$ are satisfied. This is dealt with by the intermediary property in the same way as it was done for the ranking by degree ratio in the proof of Theorem 11. (Therefore, we omit this part of the proof.)

We are left to show that the ranking by Copeland score satisfies the axioms.

$\geq^{c o p}$ satisfying anonymity is obvious.

$\geq^{c o p}$ satisfying positive responsiveness follows since a node's Copeland score is increasing if its outdegree increases, which has no positive effect for the other nodes. 
$\geq^{c o p}$ satisfying independence of irrelevant arcs follows since the Copeland score of a node only depends on the arcs with its successors and predecessors.

$\geq^{c o p}$ satisfying clone property 2 follows since $\operatorname{cop}_{i}\left(N^{\prime}, D^{\prime}\right)=\operatorname{out}_{i}\left(N^{\prime}, D^{\prime}\right)-\operatorname{in}_{i}\left(N^{\prime}, D^{\prime}\right)=\left(\operatorname{out}_{i}(N, D)+\right.$ 1)- $\left(\operatorname{in}_{i}(N, D)+1\right)=\operatorname{out}_{i}(N, D)-i n_{i}(N, D)=\operatorname{cop}_{i}(N, D)$, and $\operatorname{cop}_{j}\left(N^{\prime}, D^{\prime}\right)=\operatorname{cop}_{j}(N, D)$ for all $j \in N \backslash\{i\}$, where $\left(N^{\prime}, D^{\prime}\right)$ is as given in the axiom.

$\geq^{c o p}$ satisfying the intermediary property follows since $\operatorname{cop}_{j}\left(N^{\prime}, D^{\prime}\right)=\operatorname{cop}_{j}(N, D)$ for all $j \in N$ with $\left(N^{\prime}, D^{\prime}\right)$ as given in the axiom.

Logical independence of the axioms in Theorem 2 is shown by the following alternative ranking methods. The first two methods are the same as used to show logical independence of the axioms in Theorem 1 at the end of Section 3. The third and fourth methods are a variation of the third and fourth methods at the end of Section 3.

1. Consider the ranking method that always ranks all nodes according to their label: For every $(N, D) \in \mathcal{D}$, let

$$
i>_{(N, D)} j \Leftrightarrow i<j \text { for all } i, j \in N \text {. }
$$

This ranking method satisfies all axioms of Theorem 2 except anonymity.

2. Consider the ranking method that always ranks all nodes equally irrespective of the relations among the nodes: For every $(N, D) \in \mathcal{D}$, let

$$
i \sim_{(N, D)} j \text { for all } i, j \in N \text {. }
$$

This ranking method satisfies all axioms of Theorem 2 except positive responsiveness.

3. Consider the ranking method, where (i) all nodes that belong to a top cycle are ranked above nodes that do not belong to a top cycle, and (ii) within and outside the top set (i.e. the union of all top cycles), we rank the nodes by their Copeland score. Formally, this ranking method is given as follows: For every $(N, D) \in \mathcal{D}$, let

$$
i \geq_{(N, D)} j \Leftrightarrow\left\{\begin{array}{l}
i \in T o p(N, D) \text { and } j \in N \backslash T o p(N, D) \\
\text { or } \\
i, j \in T o p(N, D) \text { and } \operatorname{cop}_{i}(N, D) \geq \operatorname{cop}_{j}(N, D) \\
\text { or } \\
i, j \in N \backslash T o p(N, D) \text { and } \operatorname{cop}_{i}(N, D) \geq \operatorname{cop}_{j}(N, D)
\end{array}\right.
$$

where $\operatorname{Top}(N, D)$ is the top set (i.e. union of all top cycles) of $(N, D)$.

This ranking method satisfies all axioms of Theorem 2 except independence of irrelevant arcs. 
4. The ranking by degree ratio satisfies all axioms of Theorem 2 except clone property 2 (see Section 3).

5. Consider the modification of the ranking by Copeland score where we give 1 point for a strict win (i.e. a node that is a successor but not a predecessor), 0 points for a draw (i.e. a node that is a successor as well as a predecessor), and subtract just half a point for a strict loss (i.e. a node that is a predecessor but not a successor). Define

$$
\widetilde{r}_{i}(N, D)=\#\left(S_{(N, D)}(i) \backslash P_{(N, D)}(i)\right)-\frac{1}{2} \#\left(P_{(N, D)}(i) \backslash S_{(N, D)}(i)\right)
$$

For every $(N, D) \in \mathcal{D}$, let

$$
i \geq j \Leftrightarrow \widetilde{r}_{i}(N, D) \geq \widetilde{r}_{j}(N, D)
$$

This ranking method satisfies all axioms of Theorem 2 except the intermediary property.

Theorems 1 and 2 present comparable axiomatic characterizations of the rankings by degree ratio and Copeland score, respectively, that only differ in one axiom - the clone property.

\section{A modification of the ranking by degree ratio choosing strong maximal nodes}

In Section 3, we defined the degree ratio as the ratio between the outdegree and indegree after adding 1 to both degrees, avoiding dividing by a zero indegree. However, we can also simply use the outdegree divided by the indegree (without adding 1's) whenever the indegree is nonzero, and specify the ranking if there are nodes with zero indegree. An obvious way to do this is (i) to always rank strong maximal nodes, i.e., nodes with zero indegree 3 , above nodes with positive indegree, (ii) rank nodes with positive indegree by their modified degree ratio, and (iii) if there are multiple strong maximal nodes, then to rank them by outdegree. This keeps the "spirit" of the degree ratio, but in some situations ranks nodes that were never losing above those with a positive indegree.

Definition 3. The modified degree ratio of node $i \in N$ in digraph $(N, D)$ is given by

$$
\bar{r}_{i}(N, D)=\left\{\begin{array}{cl}
\frac{\operatorname{out}_{i}(N, D)}{\operatorname{in}_{i}(N, D)} & \text { if } \operatorname{in}_{i}(N, D)>0, \\
\infty & \text { if } \operatorname{in}_{i}(N, D)=0 .
\end{array}\right.
$$

Definition 4. The ranking by modified degree ratio is the ranking method given by

$$
i \geq_{(N, D)}^{m d r} j \Leftrightarrow\left\{\begin{array}{l}
\operatorname{in}_{i}(N, D)=0 \text { and } \operatorname{in}_{j}(N, D)>0 \\
o r \\
i n_{i}(N, D) \cdot \operatorname{in}_{j}(N, D)>0 \text { and } \bar{r}_{i}(N, D) \geq \bar{r}_{j}(N, D) \\
o r \\
i n_{i}(N, D)=\operatorname{in}_{j}(N, D)=0 \text { and } \operatorname{out}_{i}(N, D) \geq \text { out }_{j}(N, D) .
\end{array}\right.
$$

\footnotetext{
${ }^{3}$ A node is usually called maximal if it is not strictly defeated by any other node, i.e. if every predecessor is also a successor. We speak about a strong maximal node if it does not have any predecessor at all.
} 
The ranking by modified degree ratio being different from the ranking by Copeland score can be seen from Example 1. In this example, $\operatorname{cop}_{5}(N, D)=2>1=\operatorname{cop}_{1}(N, D)$, but according to the modified degree ratio, node 1 is ranked higher than node 5 since it has zero indegree.

As illustrated in the following example, the ranking by modified degree ratio is also different from the ranking by degree ratio.

Example 2. Consider $(N, D)$ with $N=\{1,2,3,4,5\}$ and $D=\{(3,2),(2,4),(2,5)\}$ as illustrated in Figure 6 Table 2 presents the degrees and the scores in question for every node.

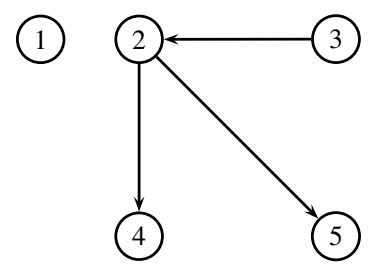

Figure 6: Example 2

\begin{tabular}{|c|c|c|c|c|}
\hline$i$ & 1 & 2 & 3 & 4,5 \\
\hline $\operatorname{out}_{i}(N, D)$ & 0 & 2 & 1 & 0 \\
\hline $\operatorname{in}_{i}(N, D)$ & 0 & 1 & 0 & 1 \\
\hline $\operatorname{cop}_{i}(N, D)$ & 0 & 1 & 1 & -1 \\
\hline$r_{i}(N, D)$ & 1 & $3 / 2$ & 2 & $1 / 2$ \\
\hline $\bar{r}_{i}(N, D)$ & $\infty$ & 2 & $\infty$ & 0 \\
\hline
\end{tabular}

Table 2: Example2

Then $r_{1}(N, D)=1<\frac{3}{2}=r_{2}(N, D)$, but according to the modified degree ratio, node 1 is ranked higher than node 2 since it has zero indegree. This example also shows that the ranking by Copeland score is different from the rankings by degree ratio and modified degree ratio: $\operatorname{cop}_{2}(N, D)=1=$ $\operatorname{cop}_{3}(N, D)$ while $r_{2}(N, D)<r_{3}(N, D)$ and node 3 is also ranked higher according to the modified degree ratio. Note that $\operatorname{in}_{1}(N, D)=\operatorname{in}_{3}(N, D)=0$, but node 3 is ranked higher than node 1 according to the modified degree ratio, since out $3(N, D)=1>0=$ out $_{1}(N, D)$.

If the degree and modified degree ratios give different ranking of two nodes with positive indegrees, then their ranking by Copeland score coincides with their ranking by degree ratio, as stated and proved by the following fact.

Fact 2. Let $\operatorname{in}_{i}(N, D) \cdot \operatorname{in}_{j}(N, D)>0$. Then, for every $i, j \in N$,

(i) If $\left[\bar{r}_{i}(N, D)<\bar{r}_{j}(N, D)\right.$ and $\left.r_{i}(N, D)>r_{j}(N, D)\right]$ then $\operatorname{cop}_{i}(N, D)>\operatorname{cop}_{j}(N, D)$.

(ii) If $\left[\bar{r}_{i}(N, D)>\bar{r}_{j}(N, D)\right.$ and $\left.r_{i}(N, D)<r_{j}(N, D)\right]$ then $\operatorname{cop}_{i}(N, D)<\operatorname{cop}_{j}(N, D)$. 
Proof

(i) Let $i n_{i}(N, D) \cdot i n_{j}(N, D)>0, \bar{r}_{i}(N, D)<\bar{r}_{j}(N, D)$ and $r_{i}(N, D)>r_{j}(N, D)$ for some $i, j \in N$. This means that $\frac{\operatorname{out}_{i}(N, D)}{i i_{i}(N, D)}<\frac{\text { out }_{j}(N, D)}{\operatorname{in}_{j}(N, D)}$ and $\frac{\operatorname{out}_{i}(N, D)+1}{\text { in }_{i}(N, D)+1}>\frac{\text { out }_{j}(N, D)+1}{\operatorname{in}_{j}(N, D)+1}$. Therefore, out $_{i}(N, D) \cdot \operatorname{in}_{j}(N, D)<$ out $_{j}(N, D)$. $\operatorname{in}_{i}(N, D)$ and $\operatorname{out}_{i}(N, D) \cdot \operatorname{in}_{j}(N, D)+\operatorname{out}_{i}(N, D)+\operatorname{in}_{j}(N, D)>\operatorname{out}_{j}(N, D) \cdot \operatorname{in}_{i}(N, D)+o u t_{j}(N, D)+\operatorname{in}_{i}(N, D)$. This implies that $\operatorname{out}_{i}(N, D)+i n_{j}(N, D)>\operatorname{out}_{j}(N, D)+i n_{i}(N, D)$, and hence $\operatorname{cop}_{i}(N, D)>\operatorname{cop}_{j}(N, D)$. (ii) It follows similarly to (i).

The ranking by modified degree ratio can be different from the ranking by degree ratio due to strong maximal nodes, but not necessarily because of that. The following example proves this observation and also illustrates Fact 2

Example 3. Consider the digraph $(N, D)$ with $N=\{1,2, \ldots, 8\}$ and $D=\{(1,2),(5,8)\} \cup\{(i, 5) \mid i=$ 3, 4, 6, 7\} as presented in Figure 7 with the corresponding Table 3

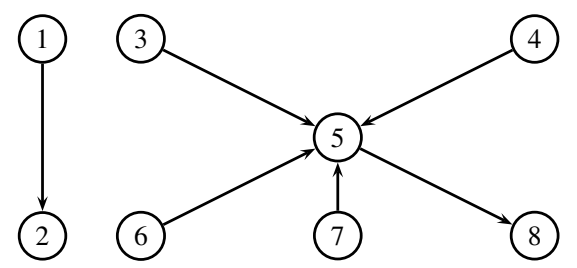

Figure 7: Example 3

\begin{tabular}{|c|c|c|c|}
\hline$i$ & $1,3,4,6,7$ & 2,8 & 5 \\
\hline $\operatorname{out}_{i}(N, D)$ & 1 & 0 & 1 \\
\hline $\operatorname{in}_{i}(N, D)$ & 0 & 1 & 4 \\
\hline $\operatorname{cop}_{i}(N, D)$ & 1 & -1 & -3 \\
\hline$r_{i}(N, D)$ & 2 & $1 / 2$ & $2 / 5$ \\
\hline $\bar{r}_{i}(N, D)$ & $\infty$ & 0 & $1 / 4$ \\
\hline
\end{tabular}

Table 3: Example 3

Note that $r_{2}(N, D)=\frac{1}{2}>\frac{2}{5}=r_{5}(N, D)$, but $\bar{r}_{2}(N, D)=0<\frac{1}{4}=\bar{r}_{5}(N, D)$. Moreover, cop ${ }_{2}(N, D)=$ $-1>-3=\operatorname{cop}_{5}(N, D)$, so the pairwise rankings between nodes 2 and 5 according to the Copeland score and degree ratio coincide.

The ranking by modified degree ratio can be axiomatized by similar axioms as before, except that we consider yet a different type of clone property, and add an axiom that explicitly requires to rank nodes with zero indegree above nodes with positive indegree. 
Clone property 2 used a subset of the arcs and nodes used in clone property 1 . Putting the arcs and nodes that are used in clone property 1 , but not in clone property 2 , we get another clone property that turns out to be satisfied by the ranking by modified degree ratio. In this clone property, new copies of the predecessors and successors of agent $i$ enter, and these new copies are related to node $i$ in the same way as their original nodes. This clone property is illustrated in Figure 8, (For transparency, the figure again only considers cases where there are no nodes that are successors as well as predecessors of node i).

Axiom 7 (Clone property 3). Let $(N, D) \in \mathcal{D}$ and $i \in N$ with $P_{D}(i) \neq \emptyset$. Let $S^{\prime}, P^{\prime} \subset \mathbb{N} \backslash N$ be such

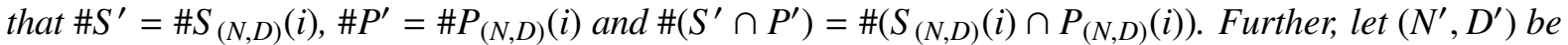
given by $N^{\prime}=N \cup S^{\prime} \cup P^{\prime}$, and

$$
D^{\prime}=D \cup\left\{(h, i) \mid h \in P^{\prime}\right\} \cup\left\{(i, h) \mid h \in S^{\prime}\right\}
$$

Then $i \geq_{(N, D)} j \Leftrightarrow i \geq_{\left(N^{\prime}, D^{\prime}\right)} j$ for all $j \in N \backslash\left(P_{(N, D)}(i) \cup S_{(N, D)}(i)\right)$.

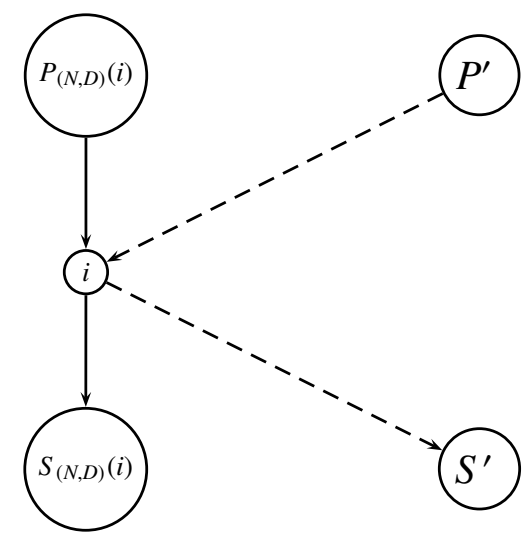

Figure 8: Axiom 7 - entrance of copies of the predecessors and successors of $i$ if $P_{(N, D)}(i) \cap S_{(N, D)}(i)=\emptyset$, the dashed arrows represent the added arcs.

Additionally, we require that we refine the set of strong maximal nodes in the sense that, if a strong maximal node exists, then the node that is ranked highest is a strong maximal node. A node is called a strong maximal node if it does not have any predecessor. Let

$$
\operatorname{SMAX}(N, D)=\left\{i \in N \mid P_{(N, D)}(i)=\emptyset\right\}
$$

be the set of strong maximal nodes of $(N, D)$.

Axiom 8 (Maximal property). If $i \in \operatorname{SMAX}(N, D)$ and $j \in N \backslash \operatorname{SMAX}(N, D)$, then $i>_{(N, D)} j$.

Replacing clone property 1 in Theorem 11 by clone property 3 , and adding the maximal property, characterizes the ranking by modified degree ratio. 
Theorem 3. The ranking by modified degree ratio is the unique ranking method satisfying anonymity, positive responsiveness, independence of irrelevant arcs, clone property 3, the intermediary property, and the maximal property.

We prove Theorem 3 in several steps that are similar to the proof of Theorem 1

Lemma 3. Let $(N, D) \in \mathcal{D}$ and $i, j \in N$ be such that $S_{(N, D)}(i) \cap P_{(N, D)}(i)=S_{(N, D)}(i) \cap P_{(N, D)}(j)=$ $S_{(N, D)}(j) \cap P_{(N, D)}(i)=S_{(N, D)}(j) \cap P_{(N, D)}(j)=\emptyset,(i, j),(j, i) \notin D$ and $i n_{i}(N, D) \cdot i_{j}(N, D)>0$. If ranking method $\geq$ satisfies the axioms of Theorem 3 then $\left[i \geq j\right.$ if and only if $\left.\bar{r}_{i}(N, D) \geq \bar{r}_{j}(N, D)\right]$.

Proor

The proof for this case goes very similar to the proof of Lemma1, but with modified definitions of the modified digraphs. We give the full proof for completeness. We distinguish the following cases:

\section{Case A}

Suppose that $\bar{r}_{i}(N, D)=\bar{r}_{j}(N, D)$. We will again shortly denote $\bar{r}_{i}$ and $\bar{r}_{j}$ for $\bar{r}_{i}(N, D)$, respectively $\bar{r}_{j}(N, D)$.

First, we enter a cloning of node $i\left(i n_{j}(N, D)-1\right.$ times), and $j\left(i n_{i}(N, D)-1\right.$ times) as in clone property 3 , in order to create symmetric positions for them.

To this end, define $P_{i}^{\prime}, P_{j}^{\prime}, S_{i}^{\prime}, S_{j}^{\prime} \subset \mathbb{N} \backslash N$ such that each pair of these sets is disjoint, and

- $\# P_{i}^{\prime}=\left(i n_{j}(N, D)-1\right) \cdot i n_{i}(N, D)$, and $\# P_{j}^{\prime}=\left(i n_{i}(N, D)-1\right) \cdot i n_{j}(N, D)$;

- $\# S_{i}^{\prime}=\left(i n_{j}(N, D)-1\right) \cdot \operatorname{out}_{i}(N, D)$, and $\# S_{j}^{\prime}=\left(i n_{i}(N, D)-1\right) \cdot \operatorname{out}_{j}(N, D)$.

Further, let digraph $\left(N^{\prime}, D^{\prime}\right) \in \mathcal{D}$ be given by

$$
N^{\prime}=N \cup S_{i}^{\prime} \cup S_{j}^{\prime} \cup P_{i}^{\prime} \cup P_{j}^{\prime}
$$

and

$$
D^{\prime}=D \cup\left(\bigcup_{h \in S_{i}^{\prime}}\{(i, h)\}\right) \cup\left(\bigcup_{h \in P_{i}^{\prime}}\{(h, i)\}\right) \cup\left(\bigcup_{h \in S_{j}^{\prime}}\{(j, h)\}\right) \cup\left(\bigcup_{h \in P_{j}^{\prime}}\{(h, j)\}\right)
$$

Next, similar to the previous proofs, we delete all arcs that do not contain node $i$ or $j$, defining $\left(N^{\prime}, D^{\prime \prime}\right)$ similar as in the previous proofs with $D^{\prime \prime}$ given by

$$
D^{\prime \prime}=\left\{(h, g) \in D^{\prime} \mid\{h, g\} \cap\{i, j\} \neq \emptyset\right\} .
$$

Notice that

(i) $\# P_{\left(N^{\prime}, D^{\prime \prime}\right)}(i)=\# P_{i}^{\prime}+\# P_{(N, D)}(i)=\left(i n_{j}(N, D)-1\right) \cdot i n_{i}(N, D)+i n_{i}(N, D)=i n_{j}(N, D) \cdot i n_{i}(N, D)=$ $\left(i n_{i}(N, D)-1\right) \cdot i n_{j}(N, D)+i n_{j}(N, D)=\# P_{j}^{\prime}+\# P_{(N, D)}(j)=\# P_{\left(N^{\prime}, D^{\prime \prime}\right)}(j)$, and 
(ii) $\# S_{\left(N^{\prime}, D^{\prime \prime}\right)}(i)=\# S_{(N, D)}(i)+\# S_{i}^{\prime}=\operatorname{out}_{i}(N, D)+\left(\operatorname{in}_{j}(N, D)-1\right) \cdot \operatorname{out}_{i}(N, D)=\operatorname{out}_{i}(N, D) \cdot \operatorname{in}_{j}(N, D)=$ out $_{j}(N, D) \cdot \operatorname{in}_{i}(N, D)=$ out $_{j}(N, D)+\left(\operatorname{in}_{i}(N, D)-1\right) \cdot$ out $_{j}(N, D)=\# S_{(N, D)}(j)+\# S_{j}^{\prime}=\# S_{\left(N^{\prime}, D^{\prime \prime}\right)}(j)$, where the fourth equality follows from $\bar{r}_{i}(N, D)=\bar{r}_{j}(N, D)$. Therefore, anonymity implies that $i \sim_{\left(N^{\prime}, D^{\prime \prime}\right)} j$. Independence of irrelevant arcs implies that the ranking between $i$ and $j$ is the same in $\left(N^{\prime}, D^{\prime \prime}\right)$ as in $\left(N^{\prime}, D^{\prime}\right)$, and thus $i \sim_{\left(N^{\prime}, D^{\prime}\right)} j$.

A repeated application of clone property 3 implies that $i \sim_{(N, D)} j$.

\section{Case B}

Suppose without loss of generality that $\bar{r}_{i}(N, D)>\bar{r}_{j}(N, D)$.

We define similar sets $S_{i}^{\prime}, S_{j}^{\prime}, P_{i}^{\prime}$ and $P_{j}^{\prime}$ as in Case A, so define $P_{i}^{\prime}, P_{j}^{\prime}, S_{i}^{\prime}, S_{j}^{\prime} \subset \mathbb{N} \backslash N$ such that each pair of these sets is disjoint, and

- $\# P_{i}^{\prime}=\left(i n_{j}(N, D)-1\right) \cdot i n_{i}(N, D)$, and $\# P_{j}^{\prime}=\left(i n_{i}(N, D)-1\right) \cdot i n_{j}(N, D)$;

- $\# S_{i}^{\prime}=\left(i n_{j}(N, D)-1\right) \cdot \operatorname{out}_{i}(N, D)$, and $\# S_{j}^{\prime}=\left(i n_{i}(N, D)-1\right) \cdot \operatorname{out}_{j}(N, D)$.

Define digraph $\left(N^{\prime}, D^{\prime}\right)$ as in Case A (see (8) and (9)).

Similar to Case A, let $D^{\prime \prime}$ be given by (10).

Similar to Case A,

(i) $\# P_{\left(N^{\prime}, D^{\prime \prime}\right)}(i)=\# P_{i}^{\prime}+\# P_{(N, D)}(i)=\left(i n_{j}(N, D)-1\right) \cdot i n_{i}(N, D)+i n_{i}(N, D)=i n_{j}(N, D) \cdot i n_{i}(N, D)=$ $\left(i n_{i}(N, D)-1\right) \cdot i n_{j}(N, D)+i n_{j}(N, D)=\# P_{j}^{\prime}+\# P_{(N, D)}(j)=\# P_{\left(N^{\prime}, D^{\prime \prime}\right)}(j)$,

but now

(ii) $\# S_{\left(N^{\prime}, D^{\prime \prime}\right)}(i)=\# S_{(N, D)}(i)+\# S_{i}^{\prime}=\operatorname{out}_{i}(N, D)+\left(\operatorname{in}_{j}(N, D)-1\right) \cdot \operatorname{out}_{i}(N, D)=\operatorname{out}_{i}(N, D) \cdot \operatorname{in}_{j}(N, D)>$ out $_{j}(N, D) \cdot \operatorname{in}_{i}(N, D)=$ out $_{j}(N, D)+\left(\right.$ in $\left._{i}(N, D)-1\right) \cdot$ out $_{j}(N, D)=\# S_{(N, D)}(j)+\# S_{j}^{\prime}=\# S_{\left(N^{\prime}, D^{\prime \prime}\right)}(j)$, where the inequality follows from $\bar{r}_{i}(N, D)>\bar{r}_{j}(N, D)$.

Let $D^{\prime \prime \prime} \subset D^{\prime \prime}$ with $\left[(h, g) \in D^{\prime \prime} \backslash D^{\prime \prime \prime} \Rightarrow h=i\right]$, and $\# S_{\left(N^{\prime}, D^{\prime \prime \prime}\right)}(i)=\# S_{\left(N^{\prime}, D^{\prime \prime \prime}\right)}(j)=\# S_{\left(N^{\prime}, D^{\prime \prime}\right)}(j)$.

Since $\left(N^{\prime}, D^{\prime \prime \prime}\right)$ is as in Case A, we have that $i \sim_{\left(N^{\prime}, D^{\prime \prime \prime}\right)} j$.

Since $D^{\prime \prime}$ is obtained from $D^{\prime \prime \prime}$ by adding arcs with node $i$ as predecessor, a repeated application of positive responsiveness implies that $i>_{\left(N^{\prime}, D^{\prime \prime}\right)} j$.

Independence of irrelevant arcs implies that the ranking between $i$ and $j$ is the same in $\left(N^{\prime}, D^{\prime}\right)$ as in $\left(N^{\prime}, D^{\prime \prime}\right)$, and thus $i>_{\left(N^{\prime}, D^{\prime}\right)} j$.

A repeated application of clone property 3 implies that $i \geq_{(N, D)} j$.

In a similar way, it can be shown that $j \nsucceq_{(N, D)} i$, and thus $i>_{(N, D)} j$. 
Lemma 4. Let $(N, D) \in \mathcal{D}$ and $i, j \in N$ be such that $S_{(N, D)}(i) \cap P_{(N, D)}(i)=S_{(N, D)}(i) \cap P_{(N, D)}(j)=$ $S_{(N, D)}(j) \cap P_{(N, D)}(i)=S_{(N, D)}(j) \cap P_{(N, D)}(j)=\emptyset,(i, j),(j, i) \notin D, \operatorname{in}_{i}(N, D)=0$ and $\operatorname{in}_{j}(N, D)>0$. If ranking method $\geq$ satisfies the axioms of Theorem 3 then $i>j$.

PROOF

This follows directly from the maximal property.

Lemma 5. Let $(N, D) \in \mathcal{D}$ and $i, j \in N$ be such that $S_{(N, D)}(i) \cap P_{(N, D)}(i)=S_{(N, D)}(i) \cap P_{(N, D)}(j)=$ $S_{(N, D)}(j) \cap P_{(N, D)}(i)=S_{(N, D)}(j) \cap P_{N, D)}(j)=\emptyset,(i, j),(j, i) \notin D, i n_{i}(N, D)=i n_{j}(N, D)=0$. If ranking method $\geq$ satisfies the axioms of Theorem 3 then $\left[i \geq j \Leftrightarrow \operatorname{out}_{i}(N, D) \geq\right.$ out $\left._{j}(N, D)\right]$.

Proof

We distinguish two cases.

\section{Case A}

Suppose that $\operatorname{in}_{i}(N, D)=i n_{j}(N, D)=0$ and $\operatorname{out}_{i}(N, D)=$ out $_{j}(N, D)$.

Let $D^{\prime} \subseteq D$ be again obtained by deleting all irrelevant arcs, so $D^{\prime}=\{(h, g) \in D \mid\{h, g\} \cap\{i, j\} \neq \emptyset\}$.

Anonymity implies that $i \sim_{\left(N, D^{\prime}\right)} j$.

Then, independence of irrelevant arcs implies that $i \sim_{(N, D)} j$.

\section{Case B}

Suppose that $i n_{i}(N, D)=i n_{j}(N, D)=0$ and out $_{i}(N, D)>\operatorname{out}_{j}(N, D)$.

Let $D^{\prime} \subseteq D$ be such that

- $(h, g) \in D \backslash D^{\prime}$ implies that $h=i$, and

- $\operatorname{out}_{i}\left(N, D^{\prime}\right)=$ out $_{j}\left(N, D^{\prime}\right)=$ out $_{j}(N, D)$.

Since $\left(N, D^{\prime}\right)$ is as in Case A, it follows that $i \sim_{\left(N, D^{\prime}\right)} j$.

A repeated application of positive responsiveness implies that $i>_{(N, D)} j$.

Proof of Theorem 3

To prove uniqueness, given Lemma's 3, 4 and 5, we still have to consider the case where not all conditions $S_{(N, D)}(i) \cap P_{(N, D)}(i)=S_{(N, D)}(i) \cap P_{N, D)}(j)=S_{(N, D)}(j) \cap P_{(N, D)}(i)=S_{(N, D)}(j) \cap P_{N, D)}(j)=\emptyset$ 
and $(i, j),(j, i) \notin D$ are satisfied. This is dealt with the intermediary property similarly to the proof of Theorem 1 .

Also, the ranking by modified degree ratio satisfying the axioms follows similarly to the proof of Theorem 1 (notice that in clone property 3 , we only consider nodes $i$ with $P_{(N, D)}(i) \neq \emptyset$ ), while $\geq^{m d r}$ satisfying the maximal property follows by definition.

The set of added arcs between new and old nodes in clone property 2 and clone property 3, form a partition of the set of added arcs between new and old nodes in clone property 1 (assuming that the added sets $S^{\prime}$ and $P^{\prime}$ in clone property 1 and 3 are the same). Hence, applying the clone property where we add the arcs between new and old nodes that underlie the ranking by degree ratio, and leaving out those that underlie the ranking by Copeland score, gives the ranking by modified degree ratio.

By Theorems 1 and 2, there is no logical relation between clone properties 1 and 2, because with the other axioms they each characterize a different ranking method. The logical relations with clone property 3 are less direct, because Theorem 3 also uses the maximal property. Obviously, clone property 3 does not imply clone property 1 since otherwise, together with the other axioms of Theorem 3)(without the maximal property), clone property 3 would characterize the ranking by degree ratio, and thus with the maximal property we have an impossibility. Similar, clone property 3 does not imply clone property 2 since otherwise, together with the other axioms of Theorem 3 (without the maximal property), clone property 3 would characterize the ranking by Copeland score, and thus with the maximal property we again have an impossibility.

Proving that clone properties 1 and 2 do not imply clone property 3 can be shown by the following example which makes clear that the ranking by degree ratio and the ranking by Copeland score do not satisfy clone property 3 .

Consider digraphs $(N, D)$ and $\left(N^{\prime}, D^{\prime}\right)$ with

(i) $i, j \in N, N \subset N^{\prime}$ with $i \neq j$ and $N^{\prime} \backslash N=\left\{i_{1}, i_{2}, i_{3}\right\}, \operatorname{in}_{i}(N, D)=\operatorname{in}_{j}(N, D)=1, \operatorname{out}_{i}(N, D)=$ $\operatorname{out}_{j}(N, D)=2$,

(ii) $D \subset D^{\prime}$ with $D^{\prime} \backslash D=\left\{\left(i_{1}, i\right),\left(i, i_{2}\right),\left(i, i_{3}\right)\right\}$.

Then $r_{i}(N, D)=r_{j}(N, D)=r_{j}\left(N^{\prime}, D^{\prime}\right)=\frac{3}{2}$ and $r_{i}\left(N^{\prime}, D^{\prime}\right)=\frac{5}{3}$. Thus $i \sim_{(N, D)}^{d r} j$ but $i>_{\left(N^{\prime}, D^{\prime}\right)}^{d r} j$, showing that the ranking by degree ratio does not satisfy clone property 3 .

Now, consider digraphs $(N, D)$ and $\left(N^{\prime}, D^{\prime}\right)$ as above. Then $\operatorname{cop}_{i}(N, D)=\operatorname{cop}_{j}(N, D)=\operatorname{cop}_{j}\left(N^{\prime}, D^{\prime}\right)=$ 
1 and $\operatorname{cop}_{i}\left(N^{\prime}, D^{\prime}\right)=2$. Thus $i \underset{(N, D)}{\sim c o p} j$ but $i>_{\left(N^{\prime}, D^{\prime}\right)}^{c o p} j$, showing that the ranking by Copeland score does not satisfy clone property 3 .

So, we conclude that the three clone properties do not imply one another.

We show logical independence of the axioms in Theorem 3 by the following alternative ranking methods.

1. Consider the ranking method that ranks all nodes without predecessors above nodes with predecessors, and within these two sets, ranks the nodes according to their label: For every $(N, D) \in \mathcal{D}$, let

$$
i \geq_{(N, D)} j \Leftrightarrow\left\{\begin{array}{l}
i n_{i}(N, D)=0 \text { and } \operatorname{in}_{j}(N, D)>0 \\
\text { or } \\
i<j \text { and } i n_{i}(N, D)=i n_{j}(N, D)=0 \\
\text { or } \\
i<j \text { and } i n_{i}(N, D) \cdot i n_{j}(N, D)>0 .
\end{array}\right.
$$

This ranking method satisfies all axioms of Theorem 3 except anonymity.

2. Consider the ranking method that ranks all nodes without predecessors above nodes with predecessors, and within these two sets ranks the nodes equally irrespective of the relations among the nodes: For every $(N, D) \in \mathcal{D}$, let

$$
i \geq_{(N, D)} j \Leftrightarrow\left\{\begin{array}{l}
\operatorname{in}_{i}(N, D)=0 \text { and } \operatorname{in}_{j}(N, D)>0 \\
\text { or } \\
\operatorname{in}_{i}(N, D)=i n_{j}(N, D)=0 \\
\text { or } \\
\operatorname{in}_{i}(N, D) \cdot i n_{j}(N, D)>0
\end{array}\right.
$$

This ranking method satisfies all axioms of Theorem 3 except positive responsiveness.

3. Consider the ranking method, where (i) nodes that have no predecessor are ranked above all nodes with predecessors, and among themselves are ranked by their outdegree, and (ii) among the nodes that have predecessors, a node $i$ is ranked higher than node $j$ if there is a directed path from $i$ to $j$ but not the other way around, or (iii) if there is a directed path in both directions or no directed path between these nodes at all then they are ranked by their modified degree ratio: For every 
$(N, D) \in \mathcal{D}$, let

$$
i \geq_{(N, D)} j \Leftrightarrow\left\{\begin{array}{l}
\operatorname{in}_{i}(N, D)=0 \text { and } i n_{j}(N, D)>0 \\
\text { or } \\
i n_{i}(N, D)=i n_{j}(N, D)=0 \text { and } \operatorname{out}_{i}(N, D) \geq \operatorname{out}_{j}(N, D) \\
\text { or } \\
i n_{i}(N, D) \cdot i n_{j}(N, D)>0,(i, j) \in \operatorname{tr}(D) \text { and }(j, i) \notin \operatorname{tr}(D) \\
\text { or } \\
i n_{i}(N, D) \cdot i n_{j}(N, D)>0,(i, j),(j, i) \in \operatorname{tr}(D) \text { and } \bar{r}_{i}(N, D) \geq \bar{r}_{j}(N, D) \\
\text { or } \\
i n_{i}(N, D) \cdot i n_{j}(N, D)>0,(i, j),(j, i) \notin \operatorname{tr}(D) \text { and } \bar{r}_{i}(N, D) \geq \bar{r}_{j}(N, D)
\end{array}\right.
$$

where $(N, \operatorname{tr}(D))$ is the transitive closure of digraph $(N, D)$. This ranking method satisfies all axioms of Theorem 1 except independence of irrelevant arcs.

4. Consider the ranking method that ranks all nodes without predecessors above nodes with predecessors, and within these two sets, ranks the nodes according to the ranking by the initial degree ratio (see Section 3): For every $(N, D) \in \mathcal{D}$, let

$$
i \geq_{(N, D)} j \Leftrightarrow\left\{\begin{array}{l}
\operatorname{in}_{i}(N, D)=0 \text { and } \operatorname{in}_{j}(N, D)>0 \\
\text { or } \\
i n_{i}(N, D)=i n_{j}(N, D)=0 \text { and } r_{i}(N, D) \geq r_{j}(N, D) \\
\text { or } \\
i n_{i}(N, D) \cdot i n_{j}(N, D)>0 \text { and } r_{i}(N, D) \geq r_{j}(N, D)
\end{array}\right.
$$

This ranking method satisfies all axioms of Theorem 3 except clone property 3 .

5. Consider a further modification of the ranking by modified degree ratio where the outdegree (respectively indegree) is modified by assigning only $\frac{1}{2}$ to every successor that is also a predecessor (respectively assigning only $\frac{1}{2}$ to every predecessor that is also a successor). Define

$$
\widehat{r}_{i}(N, D)=\frac{\#\left(S_{(N, D)}(i) \backslash P_{(N, D)}(i)\right)+\frac{1}{2} \#\left(S_{(N, D)}(i) \cap P_{(N, D)}(i)\right)}{\#\left(P_{(N, D)}(i) \backslash S_{(N, D)}(i)\right)+\frac{1}{2} \#\left(S_{(N, D)}(i) \cap P_{(N, D)}(i)\right)}
$$

Consider the ranking method that (i) ranks all nodes without predecessors above nodes with predecessors, (ii) ranks nodes without predecessors by their outdegree, and (iii) ranks nodes with 
predecessors by $\widehat{r}$. For every $(N, D) \in \mathcal{D}$, let

$$
i \geq_{(N, D)} j \Leftrightarrow\left\{\begin{array}{l}
\operatorname{in}_{i}(N, D)=0 \text { and } \operatorname{in}_{j}(N, D)>0 \\
\text { or } \\
\operatorname{in}_{i}(N, D)=i n_{j}(N, D)=0 \text { and } \operatorname{out}_{i}(N, D) \geq \operatorname{out}_{j}(N, D) \\
\text { or } \\
\operatorname{in}_{i}(N, D) \cdot \operatorname{in}_{j}(N, D)>0 \text { and } \widehat{r}_{i}(N, D) \geq \widehat{r}_{j}(N, D)
\end{array}\right.
$$

This ranking method satisfies all axioms of Theorem 3 except the intermediary property.

6. Define

$$
r_{i}^{\prime}(N, D)=\left\{\begin{array}{cc}
\bar{r}_{i}(N, D) & \text { if } \operatorname{in}_{i}(N, D)>0 \\
\operatorname{out}_{i}(N, D) & \text { if } \operatorname{in}_{i}(N, D)=0
\end{array}\right.
$$

Consider the following ranking method: For every $(N, D) \in \mathcal{D}$, let

$$
i \geq_{(N, D)} j \Leftrightarrow r_{i}^{\prime}(N, D) \geq r_{j}^{\prime}(N, D)
$$

This ranking method satisfies all axioms of Theorem 3 except the maximal property.

\section{Conclusion}

This paper considers the ranking by degree ratio and ranking by modified degree ratio, as alternatives to the famous ranking by Copeland score. Similar to the ranking by Copeland score, these rankings are based on the out- and indegree. But instead of taking the difference between the out- and indegree, we divide the outdegree by indegree. In order to avoid dividing by a zero indegree, in the degree ratio we add 1 to the out- and indegree of a node. For the modified degree ratio, we take only the out- and indegree, but by definition assume that nodes with indegree zero are ranked higher than nodes with a positive indegree. The main results of the paper are axiomatic characterizations of the rankings by degree ratio and by modified degree ratio, as well as a comparable axiomatization of the ranking by Copeland score. A main difference between these three axiomatic characterizations is in the clone property that is applied. Therefore, the axiomatizations in this paper allow us to compare the three ranking methods by their clone property.

In our view, we cannot say which clone property (and therefore ranking method) is best in general, since this depends on the application. The clone properties are some kind of invariance properties requiring that the ranking between existing nodes does not change when we clone a node. In clone property 1 , cloning means that a node is predecessor as well as successor of its copy, and also is defeating and is defeated by copies of its successors and predecessors. This seems reasonable when nodes focus 
on their 'wins' and 'losses'. This is typical in sports and game competitions, where one looks at 'who did I defeat and by whom am I defeated'. Therefore, ranking by degree ratio seems a good ranking method to apply to sports and game competitions 4

In clone property 2 , cloning a node simply means to copy this specific node, and let this clone be predecessor as well as successor of the original node. Being invariant to this type of cloning seems reasonable when one makes decisions based on pairwise comparisons. For example, when in a preference relation we introduce a new alternative that is equivalent to alternative $i$ and is not comparable with other alternatives, then this should not change the ranking of the original alternative $i$ compared to the other alternatives 5 This might explain the popularity of the ranking by Copeland score in individual and social choice theory.

Finally, our motivation to consider the ranking by modified degree ratio was more to look what happens if in cloning, we consider the arcs of cloning 1 which did not occur in cloning 2 . Is the resulting method more similar to the ranking by degree ratio or the ranking by Copeland score? It turned out that the first is the case, but we modify the ranking by putting strong maximal nodes above all other nodes (by the maximal node property), and rank the other nodes by a modified degree ratio. This seems to be rather similar to the ranking by degree ratio, but supports the idea that nodes that are not defeated (i.e. teams that lost no matches) take the highest position.

A plan for future research is to consider a degree ratio for weighted directed graphs where the links can have different weights. This allows to consider applications such as migration flows, financial flows, investment flows, etc., as mentioned in the introduction.

We conclude this paper by comparing our axioms for the ranking by Copeland score with independence of circuits 6 in [3]. From our Theorem 2 and the results in [3], it follows that, under anonymity and positive responsiveness, Bouyssou's independence of circuits is equivalent to the set of axioms [independence of irrelevant arcs, clone property 2 and the intermediary property]. Next, we verify that (without anonymity and/or positive responsiveness) these axioms do not imply one another.

\footnotetext{
${ }^{4}$ For example, participants in the popular Fortnite online video game are ranked by the 'Kill to Death ratio' (KDR), which is their degree ratio in the digraph where a player's outgoing arcs are to the opponents that he/she killed, and the ingoing arcs are from the opponents who killed him/her. In the game you can resurrect after being killed, and therefore you can be killed multiple times. The idea behind the KD ratio is that it gives the average number of kills you make on average in a life, which is considered a good measure of the strength of the players.

${ }^{5}$ Notice that, although in clone property 2 we only added the arcs between the original node $i$ and its clone, we could as well have added arcs between the clone and the predecessors and successors of the original node.

${ }^{6}$ For nonweighted digraphs as considered in this paper, independence of circuits means that the ranking of the nodes does not change if we delete or add a cycle of length 2 or 3.
} 
1. First, we show that [independence of irrelevant arcs, clone property 2 and the intermediary property] together do not imply independence of circuits.

Consider the measure wcop that assigns to every node its Copeland score multiplied by its label, i.e. $\operatorname{wcop}_{i}(N, D)=i \cdot \operatorname{cop}_{i}(N, D)=i \cdot\left(\operatorname{out}_{i}(N, D)-i n_{i}(N, D)\right)$ for all $i \in N$ and $(N, D) \in \mathcal{D}$. The corresponding (non-anonymous) ranking method is given by: For every $(N, D) \in \mathcal{D}$ and $i, j \in N$, let

$$
i \geq_{(N, D)}^{w c o p} j \Leftrightarrow \operatorname{wcop}_{i}(N, D) \geq \operatorname{wcop}_{j}(N, D)
$$

This ranking method satisfies independence of irrelevant arcs, clone property 2 and the intermediary property. It does not satisfy independence of circuits.

2. Next, we show that independence of circuits does not imply any of the axioms from the set [independence of irrelevant arcs, clone property 2 and the intermediary property].

Consider the ranking by Copeland score with partial tie-breaking rule such that, if among the nodes with equal Copeland score there is the node with the highest label in $N$, then this node is ranked strictly above the others. If this node with highest label is not amongst these nodes, then all are ranked equal. For every $(N, D) \in \mathcal{D}$, let

$$
i \geq_{(N, D)} j \Leftrightarrow\left\{\begin{array}{l}
\operatorname{cop}_{i}(N, D)>\operatorname{cop}_{j}(N, D) \\
\text { or } \\
\operatorname{cop}_{i}(N, D)=\operatorname{cop}_{j}(N, D) \text { and } i=\max _{h \in N} h \\
\text { or } \\
\operatorname{cop}_{i}(N, D)=\operatorname{cop}_{j}(N, D) \text { and } i, j \neq \max _{h \in N} h
\end{array}\right.
$$

This ranking method satisfies independence of circuits. It does not satisfy independence of irrelevant arcs, clone property 2 and the intermediary property.

\section{References}

[1] Altman, A., \& Tennenholtz, M. (2005). Ranking systems: The PageRank axioms. In EC'05 Proceedings of the 6th ACM Conference on Electronic Commerce (pp. 1-8). ACM, New York.

[2] Altman, A., \& Tennenholtz, M. (2008). Axiomatic foundations for ranking systems. Journal of Artificial Intelligence Research, 31, 473-495.

[3] Bouyssou, D. (1992). Ranking methods based on valued preference relations: A characterization of the net flow method. European Journal of Operational Research, 60, 61-67. 
[4] Bouyssou, D., \& Marchant, T. (2014). An axiomatic approach to bibliometric rankings and indices. Journal of Informetrics, 8, 449-477.

[5] Bouyssou, D., \& Marchant, T. (2018). The $\beta$-ranking and the $\beta$-measure for directed networks: Axiomatic characterizations. Social Networks, 52(1), 145-153.

[6] Bouyssou, D., \& Perny, P. (1992). Ranking methods for valued preference relations: A characterization of a method based on leaving and entering flows. European Journal of Operational Research, 61, 186-194.

[7] Brin, S., \& Page, L. (1998). The anatomy of large-scale hypertextual Web search engine. Computer Networks and ISDN Systems, 30, 107-117.

[8] de Clippel, G., Moulin, H., \& Tideman, N. (2008). Impartial division of a dollar. Journal of Economic Theory, 139, 176-191.

[9] Copeland, A. H. (1951). A Reasonable Social Welfare Function. Mimeographed Notes, University of Michigan Seminar on Applications of Mathematics to the Social Sciences.

[10] Demange, G. (2012). On the influence of a ranking system. Social Choice and Welfare, 39, 431455.

[11] Demange, G. (2014). Collective attention and ranking methods. Journal of Dynamics and Games, $1,17-43$.

[12] Demange, G. (2014). A ranking method based on handicaps. Theoretical Economics, 9(3), 915942.

[13] Demange, G. (2017). Mutual rankings. Mathematical Social Sciences, 90, 35-42.

[14] Du, Y., Lehrer, E., \& Pauzner, A. (2015). Competitive economy as a ranking device over networks. Games and Economic Behavior, 91, 1-13.

[15] Henriet, D. (1985). The Copeland choice function - an axiomatic characterization. Social Choice and Welfare, 2, 49-63.

[16] Palacios-Huerta, I., \& Volij, O. (2004). The measurement of intellectual influence. Econometrica, 72, 963-977. 
[17] Pinski, G., \& Narin, F. (1976). Citation influence for journal aggregates of scientific publications: Theory, with application to the literature of physics. Information Processing and Management, 12, 297-312.

[18] Rubinstein, A. (1980). Ranking the participants in a tournament. SIAM Journal of Applied Mathematics, 38(1), 108-111.

[19] Sen, A. K. (1979). Collective Choice and Social Welfare. North Holland Publishing Company, Amsterdam, The Netherlands.

[20] Slutzki, G., \& Volij, O. (2006). Scoring of web pages and tournaments - axiomatizations. Social Choice and Welfare, 26, 75-92.

[21] van den Brink, R., \& Gilles, R. P. (2000). Measuring domination in directed networks. Social Networks, 22, 141-157.

[22] van den Brink, R., \& Gilles, R. P. (2003). Ranking by outdegree for directed graphs. Discrete Mathematics, 271, 261-270.

[23] van den Brink, R., \& Gilles, R. P. (2009). The outflow ranking method for weighted directed graphs. European Journal of Operational Research, 193, 484-491.

[24] Woeginger, G. J. (2008). An axiomatic characterization of the Hirsch-index. Mathematical Social Sciences, 56, 224-232. 